TRANSACTIONS OF THE

AMERICAN MATHEMATICAL SOCIETY

Volume 356, Number 3, Pages 1121-1154

S 0002-9947(03)03316-6

Article electronically published on August 26, 2003

\title{
A CAPELLI HARISH-CHANDRA HOMOMORPHISM
}

\author{
TOMASZ PRZEBINDA
}

\begin{abstract}
For a real reductive dual pair the Capelli identities define a homomorphism $\mathcal{C}$ from the center of the universal enveloping algebra of the larger group to the center of the universal enveloping algebra of the smaller group. In terms of the Harish-Chandra isomorphism, this map involves a $\rho$-shift. We view a dual pair as a Lie supergroup and offer a construction of the homomorphism $\mathcal{C}$ based solely on the Harish-Chandra's radial component maps. Thus we provide a geometric interpretation of the $\rho$-shift.
\end{abstract}

\section{TABLE OF CONTEnts}

0 . Introduction

1. Ordinary classical Lie supergroups and dual pairs

2. The representations $\hat{L}_{p}$

3. The maps $\delta_{\mathfrak{g} / \mathfrak{h}}^{\prime}, \delta_{\mathfrak{g} / \mathfrak{z}}^{\prime}$

4. The $\operatorname{map} \delta_{G / H}^{\prime}$

5. The homomorphism $\mathcal{C}$

6. The oscillator representation

7. Descent to a neighborhood of a semisimple point in the symplectic space $\mathfrak{s}_{1}$

Appendix A. The Weierstrass polynomial approximation theorem

Appendix B. Completion of the proof of Theorem 2.13

References

\section{INTRODUCTION}

In this article we begin a program of investigating the character theory for a real reductive dual pair $\left(G, G^{\prime}\right)$, [H1], from a viewpoint akin to that of Harish-Chandra. We sketch the idea below.

The characters are invariant eigendistributions on the group. An invariant eigendistribution on the group is a distribution which is invariant under the conjugation by elements of the group and is an eigendistribution for the commutative algebra of the left and right invariant smooth differential operators.

The dual pair, together with the underlying symplectic space $\mathfrak{s}_{1}$, may be viewed as a classical Lie supergroup $(S, \mathfrak{s})$, where

$$
S=G \times G^{\prime}, \quad \mathfrak{s}=\mathfrak{s}_{0} \oplus \mathfrak{s}_{1}, \quad \mathfrak{s}_{0}=\mathfrak{g} \oplus \mathfrak{g}^{\prime} .
$$

Received by the editors September 4, 2002.

2000 Mathematics Subject Classification. Primary 22E46, 17B35.

This research was partially supported by NSF grant DMS 0200724. Part of the work was done while the author was visiting the Institute for Mathematical Sciences, National University of Singapore, in 2001. 
Here $\mathfrak{g}$ is the Lie algebra of $G$ and $\mathfrak{g}^{\prime}$ is the Lie algebra of $G^{\prime}$.

Let $S p$ be the symplectic group of $\mathfrak{s}_{1}$ and let $\tilde{S p} \rightarrow S p$ be the metaplectic cover. Then $\tilde{S p}$ acts on the tempered distributions on $\mathfrak{s}_{1}$ via the oscillator representation combined with the left and right twisted convolutions (section 6). Let $\tilde{S}=\tilde{G} \times \tilde{G}^{\prime}$, where $\tilde{G}$ and $\tilde{G}^{\prime}$ are the preimages of $G$ and $G^{\prime}$ in $\tilde{S p}$ via the covering map. An invariant eigendistribution on $\mathfrak{s}_{1}$ is, by definition, a distribution which is invariant under conjugation by elements of $S$ and is an eigendistribution for the algebra of the left and right $\tilde{S}$-invariant polynomial differential operators on $\mathfrak{s}_{1}$.

As shown by Howe, [H1, Theorem 1], each representation $\Pi \otimes \Pi^{\prime}$ in Howe's correspondence for the pair $\left(G, G^{\prime}\right)$ is determined by a unique (up to a scalar multiple) invariant eigendistribution $f_{\Pi \otimes \Pi^{\prime}}$ on $\mathfrak{s}_{1}$ (see [P1, section 2] for more details). Thus the distribution character $\Theta_{\Pi \otimes \Pi^{\prime}}=\Theta_{\Pi} \otimes \Theta_{\Pi^{\prime}}$ together with $f_{\Pi \otimes \Pi^{\prime}}$ forms what one is tempted to call an invariant eigendistribution on the Lie supergroup $(S, \mathfrak{s})$. Our goals are to express $f_{\Pi \otimes \Pi^{\prime}}$ in terms of $\Theta_{\Pi}$ and/or $\Theta_{\Pi^{\prime}} ; \Theta_{\Pi}$ and $\Theta_{\Pi^{\prime}}$ in terms of $f_{\Pi \otimes \Pi^{\prime}} ; \Theta_{\Pi}$ in terms of $\Theta_{\Pi^{\prime}} ;$ and $\Theta_{\Pi^{\prime}}$ in terms of $\Theta_{\Pi}$.

In a sense the algebra of the left and right $\tilde{S}$-invariant polynomial differential operators on $\mathfrak{s}_{1}$ is very well known, [H0], [H2]. However, in order to understand it from a different angle, we consider the radial component of the differential operators involved along a Cartan subspace $\mathfrak{h}_{1} \subseteq \mathfrak{s}_{1}$ (section 1 ). There are only finitely many $S$-conjugacy classes of Cartan subspaces of $\mathfrak{s}_{1}$, and every semisimple element of $\mathfrak{s}_{1}$ belongs to an $S$-orbit passing through one of them. At this point more structure is necessary.

There is a defining module $V=V_{0} \oplus V_{1}$ for $(S, \mathfrak{s})$ (section 2). Let $\mathfrak{h}_{1}^{2}$ be the subspace of $\mathfrak{s}_{0}$ spanned by all the squares $x^{2}, x \in \mathfrak{h}_{1}$. (Notice that $x^{2}$ is one half times the anticommutator of $x$ with itself.) The "miracle" is that the relation

$$
\left.\left\{\left(\left.x^{2}\right|_{V_{0}},\left.x^{2}\right|_{V_{1}}\right) ; x \in \mathfrak{h}_{1}\right\} \subseteq \mathfrak{h}_{1}^{2}\right|_{V_{0}} \times\left.\mathfrak{h}_{1}^{2}\right|_{V_{1}}
$$

is an invertible function which extends to a linear bijection

$$
\left.\left.\mathfrak{h}_{1}^{2}\right|_{V_{0}} \leftrightarrow \mathfrak{h}_{1}^{2}\right|_{V_{1}} .
$$

This bijection is one of our main reasons to introduce the notion of the Lie supergroup, rather than keep relying on the unnormalized moment maps, as in [P1]. Suppose the rank of $G=\left.S\right|_{V_{j}}$ is greater than or equal to the rank of $G^{\prime}=\left.S\right|_{V_{i}}$, $\{i, j\}=\{0,1\}$. Then $\mathfrak{h}^{\prime}=\left.\mathfrak{h}_{1}^{2}\right|_{V_{i}}$ is a Cartan subalgebra of $\mathfrak{g}^{\prime}$. By $(0.0)$, we view $\mathfrak{h}^{\prime}$ also as a subspace of $\mathfrak{g}$. At this point we need some differential operators on $\mathfrak{g}$ and on $\mathfrak{g}^{\prime}$. We get them by lifting the left regular representation of $G$ from $G$ to $\mathfrak{g}$ via the Cayley transform $c_{-}: \mathfrak{g} \rightarrow G$, by adding a twist and by taking the Fourier transform. The result is an injective algebra homomorphism from the universal enveloping algebra of $\mathfrak{g}_{\mathbb{C}}$ (the complexification of $\mathfrak{g}$ ) to the algebra of polynomial differential operators on $\mathfrak{g}$ :

$$
\hat{L}_{p}: \mathcal{U}\left(\mathfrak{g}_{\mathbb{C}}\right) \rightarrow \mathcal{P D}(\mathfrak{g})
$$

(section 2). Then we restrict the differential operators $\hat{L}_{p}(z)\left(z \in \mathcal{U}\left(\mathfrak{g}_{\mathbb{C}}\right)^{G}\right)$, from $\mathfrak{g}$ to the centralizer of $\mathfrak{h}^{\prime}$ in $\mathfrak{g}$, by Harish-Chandra's radial component map, and then to $\mathfrak{h}^{\prime}$ (if the twist is correct) by Theorem 2.13. We do the same for the group $G^{\prime}$ and get matching of differential operators on $\mathfrak{h}^{\prime}$ (Theorem 5.6). This leads to the definition (5.5) of our Capelli Harish-Chandra homomorphism

$$
\mathcal{C}: \mathcal{U}\left(\mathfrak{g}_{\mathbb{C}}\right)^{G} \rightarrow \mathcal{U}\left(\mathfrak{g}_{\mathbb{C}}^{\prime}\right)^{G^{\prime}}
$$


The existence of the homomorphism $\mathcal{C}$ follows from Theorem 7 in [H] (which we do not use in our construction). For a dual pair $\left(G, G^{\prime}\right)$, such that $\mathfrak{g}_{\mathbb{C}}=g l_{m}(\mathbb{C})$ and $\mathfrak{g}^{\prime} \mathbb{C}=g l_{n}(\mathbb{C})$, with $m=n$, the homomorphism $\mathcal{C}$ is determined by the Capelli identity, [C1, (3)]. If $m \neq n$, then [C2, Théorème VI] provides a more general identity, which determines $\mathcal{C}$. Recently Minoru Itoh proved analogous identities for the case $\mathfrak{g}_{\mathbb{C}}=o_{m}(\mathbb{C})$ and $\mathfrak{g}^{\prime} \mathbb{C}=s p_{2 n}(\mathbb{C})[\mathbb{I}$, Theorem B]. I would like to thank the referee for informing me of this work. Also, a transparent explanation of all these connections, and some generalizations, may be found in $\mathrm{H}-\mathrm{U}]$.

By dualization, $\mathcal{C}$ leads to and is determined by "a duality correspondence of infinitesimal characters" considered in [P2]. Our present approach is much more explicit. I would like to thank Wulf Rossmann for his critical and constructive remarks concerning [P2] in the Fall 1996, which in part motivated this project.

\section{Ordinary Classical Lie Supergroups and dual Pairs}

In this section we collect some simple facts which link the theory of real reductive pairs to the theory of classical Lie supergroups (see $[\bar{K}$, [Ko for the general theory of Lie superalgebras and Lie supergroups). The details and proofs will appear in [P0.

Let $\mathbb{D}=\mathbb{R}, \mathbb{C}$ or $\mathbb{H}$, and let $V_{0}, V_{1}$ be two finite-dimensional left vector spaces over $\mathbb{D}$. Set

$$
V=V_{0} \oplus V_{1}
$$

and define an element $s \in \operatorname{End}(V)$ by

$$
s\left(v_{0}+v_{1}\right)=v_{0}-v_{1} \quad\left(v_{0} \in V_{0}, v_{1} \in V_{1}\right) .
$$

Set

$$
\begin{aligned}
& \operatorname{End}(V)_{0}=\{x \in \operatorname{End}(V) ; s x=x s\}, \\
& \operatorname{End}(V)_{1}=\{x \in \operatorname{End}(V) ; s x=-x s\}, \\
& G L(V)_{0}=G L(V) \cap \operatorname{End}(V)_{0} .
\end{aligned}
$$

The real vector space $\operatorname{End}(V)_{0}$ is a Lie algebra, with the usual commutator $[x, y]=$ $x y-y x$. The adjoint action of $G L(V)_{0}$ on $\operatorname{End}(V)$,

$$
A d(g) x=g x g^{-1} \quad\left(g \in G L(V)_{0}, x \in \operatorname{End}(V)\right),
$$

preserves both $\operatorname{End}(V)_{0}$ and $\operatorname{End}(V)_{1}$. Furthermore, the anticommutator

$$
\operatorname{End}(V)_{1} \times \operatorname{End}(V)_{1} \ni(x, y) \rightarrow\{x, y\}=x y+y x \in \operatorname{End}(V)_{0}
$$

is $\mathbb{R}$-bilinear and $G L(V)_{0}$-equivariant. Set

$$
\langle x, y\rangle=\operatorname{tr}_{\mathbb{D} / \mathbb{R}}\{s x, y\} \quad(x, y \in \operatorname{End}(V)) .
$$

It is easy to see that the form (1.5) is preserved under the action of $G L(V)_{0}$.

Lemma 1.6. If $V_{0} \neq 0$ and $V_{1} \neq 0$, then the restriction of the bilinear form $\langle$, to $\operatorname{End}(V)_{1}$ is symplectic and non-degenerate. Moreover, the group homomorphism

$$
A d: G L(V)_{0} \rightarrow \operatorname{Sp}\left(\operatorname{End}(V)_{1},\langle,\rangle\right)
$$

maps the groups

$$
\begin{aligned}
& \left.G L(V)_{0}\right|_{V_{0}}=\left\{g \in G L(V)_{0} ;\left.g\right|_{V_{1}}=1\right\} \\
& \left.G L(V)_{0}\right|_{V_{1}}=\left\{g \in G L(V)_{0} ;\left.g\right|_{V_{0}}=1\right\}
\end{aligned}
$$


injectively onto an irreducible dual pair of type II in the symplectic group $\operatorname{Sp}\left(\operatorname{End}(V)_{1},\langle\rangle,\right)$.

Let $\iota$ be a possibly trivial involution on $\mathbb{D}$. Let $\phi_{0}$ be a non-degenerate $\iota$ Hermitian form on $V_{0}$, and let $\phi_{1}$ be a non-degenerate $\iota$-skew-Hermitian form on $V_{1}$. Set $\phi=\phi_{0} \oplus \phi_{1}$. Then

$$
\phi(u, v)=\iota(\phi(v, s u)) \quad(u, v \in V) .
$$

Define

$$
\begin{aligned}
& \mathfrak{s}(V, \phi)_{0}=\left\{x \in \operatorname{End}(V)_{0} ; \phi(x u, v)=\phi(u,-x v), u, v \in V\right\}, \\
& \mathfrak{s}(V, \phi)_{1}=\left\{x \in \operatorname{End}(V)_{1} ; \phi(x u, v)=\phi(u, s x v), u, v \in V\right\}, \\
& S(V, \phi)_{0}=\left\{g \in G L(V)_{0} ; \phi(g u, g v)=\phi(u, v), u, v \in V\right\} .
\end{aligned}
$$

Clearly, $S(V, \phi)_{0}$ is a Lie subgroup of $G L(V)_{0}$, with the Lie algebra $\mathfrak{s}(V, \phi)_{0}$. Moreover, it is easy to check that the anticommutator (1.4) maps $\mathfrak{s}(V, \phi)_{1} \times \mathfrak{s}(V, \phi)_{1}$ into $\mathfrak{s}(V, \phi)_{0}$. Furthermore, the adjoint action of $S(V, \phi)_{0}$ preserves both $\mathfrak{s}(V, \phi)_{0}$ and $\mathfrak{s}(V, \phi)_{1}$, and the form (1.5).

Lemma 1.9. If $V_{0} \neq 0$ and $V_{1} \neq 0$, then the restriction of the bilinear form $\langle$, to $\mathfrak{s}(V, \phi)_{1}$ is symplectic and non-degenerate. Moreover,

$$
A d: S(V, \phi)_{0} \rightarrow S p\left(\mathfrak{s}(V, \phi)_{1},\langle,\rangle\right)
$$

maps the groups

$$
\begin{aligned}
& \left.S(V, \phi)_{0}\right|_{V_{0}}=\left\{g \in S(V, \phi)_{0} ;\left.g\right|_{V_{1}}=1\right\} \\
& \left.S(V, \phi)_{0}\right|_{V_{1}}=\left\{g \in S(V, \phi)_{0} ;\left.g\right|_{V_{0}}=1\right\}
\end{aligned}
$$

injectively onto an irreducible dual pair of type $I$ in the symplectic group $\operatorname{Sp}\left(\mathfrak{s}(V, \phi)_{1},\langle\rangle,\right)$.

Definition 1.10. An irreducible ordinary classical Lie supergroup is a pair $(S, \mathfrak{s})$ with $\mathfrak{s}=\mathfrak{s}_{0} \oplus \mathfrak{s}_{1}$, where either

$$
S=S(V, \phi)_{0}, \mathfrak{s}_{0}=\mathfrak{s}(V, \phi)_{0}, \mathfrak{s}_{1}=\mathfrak{s}(V, \phi)_{1}, \text { as in (1.8), }
$$

or

$$
S=G L(V)_{0}, \mathfrak{s}_{0}=\operatorname{End}(V)_{0}, \mathfrak{s}_{1}=\operatorname{End}(V)_{1}, \text { as in (1.3). }
$$

The pair $(S, \mathfrak{s})$ is a supergroup of type I in the case (I) and of type II in the case (II). The space $V$ shall be called the defining module for $(S, \mathfrak{s})$. If needed, we shall indicate this by writing $S=S(V)$ and $\mathfrak{s}=\mathfrak{s}(V)$.

If $\left\{\left(S^{n}, \mathfrak{s}^{n}\right), n=1,2,3, \ldots, N\right\}$, is a finite collection of ordinary classical Lie supergroups, then $\left(S^{1} \times S^{2} \times \cdots \times S^{N}, \mathfrak{s}^{1} \oplus \mathfrak{s}^{2} \oplus \cdots \oplus \mathfrak{s}^{N}\right)$, with the obvious structure, is called an ordinary classical Lie supergroup.

(Here the term "ordinary" places the Lie supergroups we consider within a larger family of the classical Lie supergroups, which include the special linear Lie supergroups; see $[\mathrm{K}]$ or $[\mathrm{Ko}$.)

In order to simplify the notation we shall write $g x$ instead of $A d(g) x$, for $g \in S$ and $x \in \mathfrak{s}_{1}$, and similarly for $x^{2}$. For $x \in \mathfrak{s}_{1}$ define the anticommutant of $x$ in $\mathfrak{s}_{1}$ by

$$
{ }^{x} \mathfrak{s}_{1}=\left\{z \in \mathfrak{s}_{1} ;\{x, z\}=0\right\} .
$$


Definition 1.12. An element $x \in \mathfrak{s}_{1}$ is called semisimple if and only if $x$ is semisimple as an endomorphism of $V$. An element $x \in \mathfrak{s}_{1}$ is regular if and only if the $S$-orbit through $x$ is of maximal possible dimension. A Cartan subspace $\mathfrak{h}_{1} \subseteq \mathfrak{s}_{1}$ is the double anticommutant $\mathfrak{h}_{1}={ }^{\left({ }^{x} \mathfrak{s}_{1}\right)} \mathfrak{s}_{1}$ of a regular semisimple element $x \in \mathfrak{s}_{1}$.

Proposition 1.13. If $x \in \mathfrak{s}_{1}$ is semisimple, then so is $x^{2} \in \mathfrak{s}_{0}$. The map

$$
\mathfrak{s}_{1} \supseteq S x \rightarrow S x^{2} \subseteq \mathfrak{s}_{0}
$$

is injective on the set of semisimple orbits in $\mathfrak{s}_{1}$. The set of regular semisimple elements is dense in $\mathfrak{s}_{1}$.

There are finitely many $S$-conjugacy classes of Cartan subspaces in $\mathfrak{s}_{1}$. Every semisimple element of $\mathfrak{s}_{1}$ belongs to the $A d(S)$-orbit through an element of a Cartan subspace. Any two elements of a Cartan subspace $\mathfrak{h}_{1} \subseteq \mathfrak{s}_{1}$ commute in End $(V)$.

The complexification $\left(S_{\mathbb{C}}, \mathfrak{s}_{\mathbb{C}}\right)$ is a Lie supergroup, and all Cartan subspaces in $\mathfrak{s}_{1, \mathbb{C}}$ are $S_{\mathbb{C}}$-conjugate.

Let $\mathfrak{h}_{1} \subseteq \mathfrak{s}_{1}$ be a Cartan subspace and let $\mathfrak{h}_{1}^{\text {reg }} \subseteq \mathfrak{h}_{1}$ be the subset of regular elements. Denote by $\mathfrak{h}_{1}^{2}=\left\{\mathfrak{h}_{1}, \mathfrak{h}_{1}\right\}$ the linear span of the elements $\{x, y\}$, where $x, y \in \mathfrak{h}_{1}$. (This is the same as the span of all the squares, $x^{2}=\frac{1}{2}\{x, x\}, x \in \mathfrak{s}_{1}$.)

Proposition 1.14. The relation

$$
\left.\left\{\left(\left.x^{2}\right|_{V_{0}},\left.x^{2}\right|_{V_{1}}\right) ; x \in \mathfrak{h}_{1}\right\} \subseteq \mathfrak{h}_{1}^{2}\right|_{V_{0}} \times\left.\mathfrak{h}_{1}^{2}\right|_{V_{1}}
$$

is an invertible function, which extends uniquely to a linear bijection

$$
\left.\left.\mathfrak{h}_{1}^{2}\right|_{V_{0}} \leftrightarrow \mathfrak{h}_{1}^{2}\right|_{V_{1}} .
$$

Suppose the rank of $\left.S\right|_{V_{i}}$ is less than or equal to the rank of $\left.S\right|_{V_{j}},\{i, j\}=\{0,1\}$. Then $\left.\mathfrak{h}_{1}^{2}\right|_{V_{i}}$ is a Cartan subalgebra of $\left.\mathfrak{s}_{0}\right|_{V_{i}}$.

Set $V^{0}=\left\{v \in V ; x v=0\right.$, for all $\left.x \in \mathfrak{h}_{1}\right\}, V^{1}=\left\{x v \in V ; x \in \mathfrak{h}_{1}, v \in V\right\}$, $V_{j}^{0}=V^{0} \cap V_{j}$ and $V_{j}^{1}=V^{1} \cap V_{j}$. Then

$$
V_{j}=V_{j}^{0} \oplus V_{j}^{1}
$$

and the sum is orthogonal in the type I case. The centralizer of $\left.\mathfrak{h}_{1}^{2}\right|_{V_{j}}$ in $\left.\mathfrak{s}_{0}\right|_{V_{j}}$ is equal to

$$
\mathfrak{z}_{\mathfrak{s}_{0}\left(V_{j}\right)}\left(\left.\mathfrak{h}_{1}^{2}\right|_{V_{j}}\right)=\left.\left.\mathfrak{h}_{1}^{2}\right|_{V_{j}^{1}} \oplus \mathfrak{s}_{0}\left(V^{0}\right)\right|_{V_{j}^{0}},
$$

where $\left.\mathfrak{h}_{1}^{2}\right|_{V_{j}^{1}}$ is isomorphic to $\left.\mathfrak{h}_{1}^{2}\right|_{V_{j}}$ by the restriction from $V_{j}$ to $V_{j}^{1}$. Under the identification (b), the normalizer of $\left.\mathfrak{h}_{1}^{2}\right|_{V_{j}^{1}}$ in $\left.S\left(V^{1}\right)\right|_{V_{j}^{1}}$ is isomorphic to the normalizer of $\left.\mathfrak{h}_{1}^{2}\right|_{V_{i}}$ in $\left.S\right|_{V_{i}}$ :

$$
N_{\left.S\left(V^{1}\right)\right|_{V_{j}^{1}}}\left(\left.\mathfrak{h}_{1}^{2}\right|_{V_{j}^{1}}\right)=N_{\left.S\right|_{V_{i}}}\left(\left.\mathfrak{h}_{1}^{2}\right|_{V_{i}}\right) .
$$

The normalizer of $\mathfrak{z}_{\mathfrak{s}_{0}\left(V_{j}\right)}\left(\left.\mathfrak{h}_{1}^{2}\right|_{V_{j}}\right)$ in $\left.S\right|_{V_{j}}$ preserves the decomposition (c) and is isomorphic to the direct product

$$
N_{\left.S\right|_{V_{j}}}\left(\mathfrak{z}_{\mathfrak{s}_{0}\left(V_{j}\right)}\left(\left.\mathfrak{h}_{1}^{2}\right|_{V_{j}}\right)\right)=N_{\left.S\right|_{V_{i}}}\left(\left.\mathfrak{h}_{1}^{2}\right|_{V_{i}}\right) \times\left. S\left(V^{0}\right)\right|_{V_{j}^{0}} .
$$

In order to compress the notation we shall write

$$
\begin{aligned}
G & =\left.S\right|_{V_{j}}, \mathfrak{g}=\left.\mathfrak{s}_{0}\right|_{V_{j}}, G^{\prime}=\left.S\right|_{V_{i}}, \mathfrak{g}^{\prime}=\left.\mathfrak{s}_{0}\right|_{V_{i}}, \\
\mathfrak{h}^{\prime} & =\left.\mathfrak{h}_{1}^{2}\right|_{V_{i}}=\left.\mathfrak{h}_{1}^{2}\right|_{V_{j}^{1}}, \mathfrak{z}=\mathfrak{z}_{\mathfrak{s}_{0}\left(V_{j}\right)}\left(\left.\mathfrak{h}_{1}^{2}\right|_{V_{j}}\right), \mathfrak{z}^{\prime \prime}=\left.s_{0}\left(V^{0}\right)\right|_{V_{j}^{0}}, \\
Z & =N_{\left.S\right|_{V_{j}}}\left(\mathfrak{z}_{\mathfrak{s}_{0}\left(V_{j}\right)}\left(\left.\mathfrak{h}_{1}^{2}\right|_{V_{j}}\right)\right), W^{\prime}=N_{\left.S\right|_{V_{i}}}\left(\left.\mathfrak{h}_{1}^{2}\right|_{V_{i}}\right), Z^{\prime \prime}=\left.S\left(V^{0}\right)\right|_{V_{j}^{0}} .
\end{aligned}
$$


Also let

$$
\begin{gathered}
\tau_{\mathfrak{g}}:\left.\mathfrak{s}_{1} \ni x \rightarrow x^{2}\right|_{V_{j}} \in \mathfrak{g}, \quad d=\operatorname{dim}_{\mathbb{D}}\left(V_{j}\right), \\
\tau_{\mathfrak{g}^{\prime}}:\left.\mathfrak{s}_{1} \ni x \rightarrow x^{2}\right|_{V_{i}} \in \mathfrak{g}^{\prime}, \quad d^{\prime}=\operatorname{dim}_{\mathbb{D}}\left(V_{i}\right) .
\end{gathered}
$$

Then for $x \in \mathfrak{s}_{1}$,

$$
\langle y x, x\rangle=\left\{\begin{array}{l}
(-1)^{j} 2 \operatorname{tr}_{\mathbb{D} / \mathbb{R}}\left(y \tau_{\mathfrak{g}}(x)\right) \quad \text { if } \quad y \in \mathfrak{g}, \\
(-1)^{i} 2 \operatorname{tr}_{\mathbb{D} / \mathbb{R}}\left(y \tau_{\mathfrak{g}^{\prime}}(x)\right) \quad \text { if } \quad y \in \mathfrak{g}^{\prime} .
\end{array}\right.
$$

\section{The representations $\hat{L}_{p}$}

Recall the Cayley transform

$$
c(x)=(x+1)(x-1)^{-1} \quad(x \in \operatorname{End}(V), \quad x-1 \text { invertible }) .
$$

Let $\mathfrak{g}^{c}, G^{c}$ denote the domain of the Cayley transform $c$ in $\mathfrak{g}$ and in $G^{c}$, respectively. Then the maps

$$
c: \mathfrak{g}^{c} \rightarrow G^{c}, \quad c: G^{c} \rightarrow \mathfrak{g}^{c}
$$

are bijections and the compositions

$$
c^{2}: \mathfrak{g}^{c} \rightarrow \mathfrak{g}^{c}, \quad c^{2}: G^{c} \rightarrow G^{c}
$$

are the identities on the corresponding sets. Furthermore,

$$
c(0)=-1, \quad c(-x)=c(x)^{-1} \quad\left(x \in \mathfrak{g}^{c}\right),
$$

and for $y \in \mathfrak{g}^{c}$ with $x+y$ invertible in $\operatorname{End}\left(V_{j}\right)$ (see (1.15) for $V_{j}$ ),

$$
c(c(x) c(y))=(y-1)(x+y)^{-1}(x-1)+1
$$

(see [H2, (10.2.3)]). Let

$$
c_{-}(x)=-c(x)=(1+x)(1-x)^{-1} .
$$

Then $c_{-}(0)=1$ and the maps

$$
c_{-}: \mathfrak{g}^{c} \rightarrow-G^{c}, \quad c_{-}: G^{c} \rightarrow-\mathfrak{g}^{c}
$$

are bijections. A straightforward computation using the above formulas shows that the derivative of the map

$$
\mathfrak{g} \ni x \rightarrow c_{-}^{-1}\left(c_{-}(x)^{-1}\left(c_{-}(y)\right) \in \mathfrak{g}\right.
$$

at $x=0$ coincides with the following linear map:

$$
\mathfrak{g} \ni x \rightarrow(y-1) x(y+1) \in \mathfrak{g} .
$$

Recall the left regular representation of $\mathfrak{g}$ :

$$
L(x) \Psi(g)=\left.\frac{d}{d t} \Psi(\exp (-t x) g)\right|_{t=0} \quad\left(x \in \mathfrak{g}, g \in G, \Psi \in C^{\infty}(G)\right) .
$$

Let

$$
c_{-}^{*}(L(x)) \psi=\left(L(x)\left(\psi \circ c_{-}^{-1}\right)\right) \circ c_{-} \quad\left(\psi \in C_{c}^{\infty}\left(\mathfrak{g}^{c}\right), x \in \mathfrak{g}\right) .
$$

Since the derivative of $c_{-}$at zero is two times the identity, the above formula may be rewritten as

$$
c_{-}^{*}(L(x)) \psi(y)=\left.\frac{1}{2} \frac{d}{d t} \psi\left(c_{-}^{-1}\left(c_{-}(t x)^{-1} c_{-}(y)\right)\right)\right|_{t=0} .
$$

Then (2.1) implies that

$$
c_{-}^{*}(L(x))=\partial\left(\frac{1}{2}(y-1) x(y+1)\right) \quad(x, y \in \mathfrak{g}),
$$


where

$$
\partial(z) \psi(y)=\left.\frac{d}{d t} \psi(y+t z)\right|_{t=0} \quad\left(z \in \mathfrak{g}, \psi \in C^{\infty}(\mathfrak{g})\right)
$$

Let $\mathcal{S}\left(\mathfrak{g}_{\mathbb{C}}\right)$ denote the symmetric algebra of the vector space $\mathfrak{g}_{\mathbb{C}}$. Let $\mathcal{S}^{d}\left(\mathfrak{g}_{\mathbb{C}}\right)$ be the subspace of all the homogeneous elements of degree $d=0,1,2, \ldots$, and let $\mathcal{S}_{d}\left(\mathfrak{g}_{\mathbb{C}}\right)=\sum_{e=0}^{d} \mathcal{S}^{e}\left(\mathfrak{g}_{\mathbb{C}}\right)$. We shall say that a function

$$
q: \mathfrak{g} \rightarrow \mathcal{S}\left(\mathfrak{g}_{\mathbb{C}}\right)
$$

is analytic, polynomial or rational if there is a non-negative integer $d$ such that the range of the function $q$ is contained in $\mathcal{S}_{d}\left(\mathfrak{g}_{\mathbb{C}}\right)$, and the function

$$
q: \mathfrak{g} \rightarrow \mathcal{S}_{d}\left(\mathfrak{g}_{\mathbb{C}}\right)
$$

is analytic, polynomial or rational, respectively. For $q$ as in (2.5) let $\partial \circ q$ denote the differential operator on $\mathfrak{g}$ defined by

$$
\partial \circ q \psi(y)=\partial(q(y)) \psi(y) \quad\left(\psi \in C^{\infty}(\mathfrak{g}), y \in \mathfrak{g}\right) .
$$

The differential operator $\partial \circ q$ is called analytic, polynomial or rational if and only if the function $q$ has the indicated property. For each $y \in \mathfrak{g}, q(y)$ is called the local expression of the differential operator $\partial \circ q$ at $y$. Let $\mathcal{P} \mathcal{D}(\mathfrak{g})$ denote the algebra of polynomial differential operators on $\mathfrak{g}$. For more details see [H-C3, section 3].

Implicitly, formula (2.3) says that the differential operator $c_{-}^{*}(L(x))$, originally defined on $\mathfrak{g}^{c}$, extends to a polynomial differential operator (in fact a vector field) on $\mathfrak{g}$. The local expression of $c_{-}^{*}(L(x))$ at $y \in \mathfrak{g}$ is $\frac{1}{2}(y-1) x(y+1)$.

Let $B(x, y)(x, y \in \mathfrak{g})$ be any non-degenerate symmetric $A d(G)$-invariant bi-linear form on $\mathfrak{g}$. The map

$$
\mathfrak{g} \ni x \rightarrow B(x,) \in \mathfrak{g}^{*}
$$

extends uniquely to an algebra isomorphism from $\mathcal{S}\left(\mathfrak{g}_{\mathbb{C}}\right)$ onto the algebra $\mathcal{P}\left(\mathfrak{g}_{\mathbb{C}}\right)$ of complex-valued polynomial functions on $\mathfrak{g}_{\mathbb{C}}$, and thus provides an identification $\mathcal{S}\left(\mathfrak{g}_{\mathbb{C}}\right)=\mathcal{P}\left(\mathfrak{g}_{\mathbb{C}}\right)$. We identify $\mathcal{P}\left(\mathfrak{g}_{\mathbb{C}}\right)$ with $\mathcal{P}(\mathfrak{g})$ by restriction from $\mathfrak{g}_{\mathbb{C}}$ to $\mathfrak{g}$.

For $p \in \mathbb{C}$ let

$$
\tilde{p}= \begin{cases}\frac{1}{2} p & \text { if } G \text { is an isometry group } \\ p & \text { if } G \text { is a general linear group. }\end{cases}
$$

Set

$$
L_{p}(x)=c_{-}^{*}(L(x))+\tilde{p} x \quad(x \in \mathfrak{g}),
$$

where the second $x$ on the right hand side is viewed as the multiplication operator

$$
\psi(y) \rightarrow B(x, y) \psi(y)
$$

A straightforward computation shows that

$$
L_{p}([x, y])=\left[L_{p}(x), L_{p}(y)\right] \quad(x, y \in \mathfrak{g}),
$$

where the commutator on the right hand side is taken in $\mathcal{P} \mathcal{D}(\mathfrak{g})$. We identify $\mathcal{P} \mathcal{D}(\mathfrak{g})$ with $\mathcal{P} \mathcal{D}\left(\mathfrak{g}_{\mathbb{C}}\right)$ via the restriction of the local expression of the differential operators from $\mathfrak{g}_{\mathbb{C}}$ to $\mathfrak{g}$. 
We normalize the Lebesgue measure $d y$ on $\mathfrak{g}$ so that the Fourier transform and the inverse Fourier transform of a Schwartz function $\psi$ be given by

$$
\begin{aligned}
& \mathcal{F} \psi(z)=\int_{\mathfrak{g}} \psi(y) e^{-i B(y, z)} d y, \\
& \mathcal{F}^{-1} \psi(z)=\int_{\mathfrak{g}} \psi(y) e^{i B(y, z)} d y .
\end{aligned}
$$

Then, for $x \in \mathfrak{g}$,

$$
\begin{gathered}
\mathcal{F} \partial(x) \mathcal{F}^{-1} \psi(y)=i B(x, y) \psi(y), \\
\mathcal{F} B(x,) \mathcal{F}^{-1} \psi(y)=i \partial(x) \psi(y) .
\end{gathered}
$$

Since the algebra $\mathcal{P} \mathcal{D}(\mathfrak{g})$ is generated by $\mathcal{S}\left(\mathfrak{g}_{\mathbb{C}}\right)$ and $\partial\left(\mathcal{S}\left(\mathfrak{g}_{\mathbb{C}}\right)\right)$, the conjugation by $\mathcal{F}$ preserves $\mathcal{P D}(\mathfrak{g})$. For $D \in \mathcal{P} \mathcal{D}(\mathfrak{g})$, let $\hat{D}=\mathcal{F} D \mathcal{F}^{-1}$. Then, under our identification $\mathcal{S}\left(\mathfrak{g}_{\mathbb{C}}\right)=\mathcal{P}\left(\mathfrak{g}_{\mathbb{C}}\right),(2.9)$ may be rewritten in a more familiar form as follows:

$$
\begin{aligned}
& \partial(x)^{\wedge}=i x, \\
& \hat{x}=i \partial(x) .
\end{aligned}
$$

Define

$$
\hat{L}_{p}(x)=L_{p}(x)^{r} \quad(x \in \mathfrak{g}) .
$$

Then $\hat{L}_{p}$ extends to an algebra homomorphism

$$
\hat{L}_{p}: \mathcal{U}\left(\mathfrak{g}_{\mathbb{C}}\right) \rightarrow \mathcal{P} \mathcal{D}\left(\mathfrak{g}_{\mathbb{C}}\right) .
$$

In particular, $\hat{L}_{p}$ may be viewed as a representation of $\mathfrak{g}$ on $\mathcal{P}\left(\mathfrak{g}_{\mathbb{C}}\right)$. In order to indicate that $\hat{L}_{p}$ is a representation of $\mathfrak{g}$, we shall sometimes denote it by $\hat{L}_{\mathfrak{g}, p}$. As in [P1, (4.10)], let

$$
r_{\mathfrak{g}}= \begin{cases}2 \operatorname{dim}_{\mathbb{R}}(\mathfrak{g}) / \operatorname{dim}_{\mathbb{R}}\left(V_{j}\right) & \text { if } G \text { is an isometry group, } \\ \operatorname{dim}_{\mathbb{R}}(\mathfrak{g}) / \operatorname{dim}_{\mathbb{R}}\left(V_{j}\right) & \text { if } G \text { is a general linear group. }\end{cases}
$$

Let $d^{\prime}=\operatorname{dim}_{\mathbb{D}}\left(V_{i}\right)$, as in (1.16).

Theorem 2.13. The ideal $\mathbf{I}\left(\tau_{\mathfrak{g}}\left(\mathfrak{s}_{1}\right)\right) \subseteq \mathcal{P}\left(\mathfrak{g}_{\mathbb{C}}\right)$ of polynomials vanishing on $\tau_{\mathfrak{g}}\left(\mathfrak{s}_{1}\right)$ is $\hat{L}_{p}(\mathfrak{g})$-invariant if and only if $p=r_{\mathfrak{g}}-d^{\prime} / 2$.

In particular, $\hat{L}_{r_{\mathfrak{g}}-d^{\prime} / 2}$ defines a representation of $\mathfrak{g}$ on the quotient space $\mathcal{P}\left(\mathfrak{g}_{\mathbb{C}}\right) / \mathbf{I}\left(\tau\left(s_{1}\right)\right)$. More precisely, $\hat{L}_{r_{\mathfrak{g}}-d^{\prime} / 2}$ induces an algebra homomorphism from $\mathcal{U}\left(\mathfrak{g}_{\mathbb{C}}\right)$ to the algebra of differential operators on $\tau_{\mathfrak{g}}\left(\mathfrak{s}_{1, \mathbb{C}}\right)$, the Zariski closure of $\tau_{\mathfrak{g}}\left(s_{1}\right)$ in $\mathfrak{g}_{\mathbb{C}}$ (see [Sm-St] for the definition of a differential operator on a variety).

In particular, the ideal $\mathbf{I}(0) \subseteq \mathcal{P}\left(\mathfrak{g}_{\mathbb{C}}\right)$ of polynomials vanishing at $0 \in \mathfrak{g}_{\mathbb{C}}$ is $\hat{L}_{p}(\mathfrak{g})$-invariant if and only if $p=r_{\mathfrak{g}}$. Thus there is an algebra homomorphism

$$
\varepsilon_{\mathfrak{g}}: \mathcal{U}\left(\mathfrak{g}_{\mathbb{C}}\right) \rightarrow \mathbb{C}
$$

such that for any $\psi \in \mathcal{P}\left(\mathfrak{g}_{\mathbb{C}}\right)$ and any $z \in \mathcal{U}\left(\mathfrak{g}_{\mathbb{C}}\right)$,

$$
\hat{L}_{r_{\mathfrak{g}}}(z) \psi(0)=\varepsilon_{\mathfrak{g}}(z) \psi(0) .
$$

The map $\varepsilon_{\mathfrak{g}}$ coincides with the algebra homomorphism by which $\mathcal{U}\left(\mathfrak{g}_{\mathbb{C}}\right)$ acts on the trivial representation. 
There is an overlap between Theorem 2.13 and the formulas [L-S1 pp. 69-70] of Levasseur and Stafford. Our construction relates some of these formulas to the left regular representation of $G$.

Proof. Suppose the Lie superalgebra $\mathfrak{s}$ is complex, i.e. $\mathbb{D}=\mathbb{C}$ and $\iota=1$. Then there is a real Lie superalgebra $\tilde{\mathfrak{s}}(\mathbb{D}=\mathbb{R})$ such that $\tilde{\mathfrak{s}}_{\mathbb{C}}=\mathfrak{s}$, with the defining module $\tilde{V}$. In particular (see (1.16)),

$$
\mathfrak{g}=\left.\mathfrak{s}_{0}\right|_{V_{j}}=\left(\left.\tilde{\mathfrak{s}}_{0}\right|_{\tilde{V}_{j}}\right)_{\mathbb{C}}=\tilde{\mathfrak{g}}_{\mathbb{C}} .
$$

Let $\tilde{d}=\operatorname{dim}_{\mathbb{R}} \tilde{V}_{i}$. Then $\tilde{d}^{\prime}=d\left(=\operatorname{dim}_{\mathbb{C}}, V_{i}\right)$. Furthermore, $r_{\mathfrak{g}}=r_{\tilde{\mathfrak{g}}}$, so that

$$
r_{\mathfrak{g}}-d^{\prime} / 2=r_{\tilde{\mathfrak{g}}}-\tilde{d}^{\prime} / 2 \text {. }
$$

Moreover,

$$
\begin{aligned}
& \mathfrak{g}_{\mathbb{C}}=\mathfrak{g} \oplus \mathfrak{g}=\tilde{\mathfrak{g}}_{\mathbb{C}} \oplus \tilde{\mathfrak{g}}_{\mathbb{C}}, \\
& \tau_{\mathfrak{g}}\left(\mathfrak{s}_{1, \mathbb{C}}\right)=\tau_{\mathfrak{g}}\left(\mathfrak{s}_{1}\right) \times \tau_{\mathfrak{g}}\left(\mathfrak{s}_{1}\right)=\tau_{\tilde{\mathfrak{g}}}\left(\tilde{\mathfrak{s}}_{1, \mathbb{C}}\right) \times \tau_{\tilde{\mathfrak{g}}}\left(\tilde{\mathfrak{s}}_{1, \mathbb{C}}\right), \\
& \mathcal{U}\left(\mathfrak{g}_{\mathbb{C}}\right)=\mathcal{U}\left(\tilde{\mathfrak{g}}_{\mathbb{C}}\right) \otimes \mathcal{U}\left(\tilde{\mathfrak{g}}_{\mathbb{C}}\right), \\
& \mathcal{P}\left(\mathfrak{g}_{\mathbb{C}}\right)=\mathcal{P}\left(\tilde{\mathfrak{g}}_{\mathbb{C}}\right) \otimes \mathcal{P}\left(\tilde{\mathfrak{g}}_{\mathbb{C}}\right), \\
& \mathcal{P} \mathcal{D}\left(\mathfrak{g}_{\mathbb{C}}\right)=\mathcal{P} \mathcal{D}\left(\tilde{\mathfrak{g}}_{\mathbb{C}}\right) \otimes \mathcal{P} \mathcal{D}\left(\tilde{\mathfrak{g}}_{\mathbb{C}}\right), \\
& \hat{L}_{\mathfrak{g}, p}=\hat{L}_{\tilde{\mathfrak{g}}, p} \otimes \hat{L}_{\tilde{\mathfrak{g}}, p} \quad(p \in \mathbb{C}) .
\end{aligned}
$$

Thus it is clear that in order to prove the theorem we may assume that $(\mathbb{D}, \iota) \neq$ $(\mathbb{C}, 1)$. The last statement of the theorem will be verified in Appendix B.

Let $\left\{e_{\alpha} ; \alpha \in \mathcal{A}\right\}$ be a basis of $\mathfrak{g}$, viewed as a real vector space, and let $\left\{\check{e}_{\alpha} ; \alpha \in \mathcal{A}\right\}$ be the dual basis with respect to the form $B$ :

$$
B\left(e_{\alpha}, \check{e}_{\beta}\right)=\delta_{\alpha, \beta} \quad(\alpha, \beta \in \mathcal{A}) .
$$

For $x, y \in \mathfrak{g} \subseteq \operatorname{End}(V)$ we have

$$
(y-1) x(y+1)=y x y-[x, y]-x .
$$

In what follows we shall view $x$ as a fixed element of $\mathfrak{g}$ and $y$, as the variable. (In particular, $B(y, x)$ will be viewed as the multiplication operator $\psi(y) \rightarrow B(y, x) \psi(y)$.) Since the Fourier transform $\mathcal{F},(2.8)$, commutes with the adjoint action and since $\partial([x, y])$ is the adjoint action, we have

$$
(\partial((y-1) x(y+1)))^{\wedge}=(\partial(y x y))^{\wedge}-\partial([x, y])-(\partial(x))^{\wedge}
$$

Furthermore,

$$
\begin{aligned}
y x y & =\sum_{\alpha, \beta} B\left(y, \check{e}_{\alpha}\right) B\left(y, \check{e}_{\beta}\right) e_{\alpha} x e_{\beta} \\
& =\sum_{\alpha, \beta} B\left(y, \check{e}_{\alpha}\right) B\left(y, \check{e}_{\beta}\right) \frac{1}{2}\left(e_{\alpha} x e_{\beta}+e_{\beta} x e_{\alpha}\right),
\end{aligned}
$$

where $e_{\alpha} x e_{\beta}+e_{\beta} x e_{\alpha} \in \mathfrak{g}$, so that, by (2.10),

$$
\begin{aligned}
(\partial(y x y))^{\kappa} & =-i \sum_{\alpha, \beta} \partial\left(\check{e}_{\alpha}\right) \partial\left(\check{e}_{\beta}\right) B\left(y, \frac{1}{2}\left(e_{\alpha} x e_{\beta}+e_{\beta} x e_{\alpha}\right)\right) \\
& =-i \sum_{\alpha, \beta} \partial\left(\check{e}_{\alpha}\right) \partial\left(\check{e}_{\beta}\right) B\left(e_{\alpha} x e_{\beta}, y\right)
\end{aligned}
$$


which, by the canonical commutation relations, is equal to

$$
\begin{aligned}
& -i \sum_{\alpha, \beta} B\left(e_{\alpha} x e_{\beta}, y\right) \partial\left(\check{e}_{\alpha}\right) \partial\left(\check{e}_{\beta}\right) \\
& -i\left(\sum_{\alpha, \beta} B\left(\check{e}_{\alpha}, e_{\alpha} x e_{\beta}\right) \partial\left(\check{e}_{\beta}\right)+\sum_{\alpha, \beta} B\left(\check{e}_{\beta}, e_{\alpha} x e_{\beta}\right) \partial\left(\check{e}_{\alpha}\right)\right) .
\end{aligned}
$$

Since

$$
\begin{aligned}
& \sum_{\alpha, \beta} B\left(\check{e}_{\alpha}, e_{\alpha} x e_{\beta}\right) \partial\left(\check{e}_{\beta}\right)+\sum_{\alpha, \beta} B\left(\check{e}_{\beta}, e_{\alpha} x e_{\beta}\right) \partial\left(\check{e}_{\alpha}\right) \\
& =\sum_{\alpha, \beta} B\left(\check{e}_{\alpha}, e_{\alpha} x e_{\beta}+e_{\beta} x e_{\alpha}\right) \partial\left(\check{e}_{\beta}\right) \\
& =\sum_{\beta} B\left(e_{\beta}, \sum_{\alpha}\left(x e_{\alpha} \check{e}_{\alpha}+\check{e}_{\alpha} e_{\alpha} x\right)\right) \partial\left(\check{e}_{\beta}\right) \\
& =\partial\left(\sum_{\alpha}\left(x e_{\alpha} \check{e}_{\alpha}+\check{e}_{\alpha} e_{\alpha} x\right)\right)
\end{aligned}
$$

(where the second equality follows from the fact that $B$ is a constant multiple of the trace form on $\mathfrak{g}$ ), the above computations show that

$$
\partial(y x y)^{\wedge}=-i \sum_{\alpha, \beta} B\left(e_{\alpha} x e_{\beta}, y\right) \partial\left(\check{e}_{\alpha}\right) \partial\left(\check{e}_{\beta}\right)-i \partial\left(\sum_{\alpha}\left(x e_{\alpha} \check{e}_{\alpha}+\check{e}_{\alpha} e_{\alpha} x\right)\right) .
$$

By combining (2.3), (2.10), (2.14) and (2.15) we see that

$$
\begin{aligned}
c_{-}^{*}(L(x))=-\frac{1}{2} & \left(i \sum_{\alpha, \beta} B\left(e_{\alpha} x e_{\beta}, y\right) \partial\left(\check{e}_{\alpha}\right) \partial\left(\check{e}_{\beta}\right)\right. \\
& \left.+i \partial\left(\sum_{\alpha}\left(x e_{\alpha} \check{e}_{\alpha}+\check{e}_{\alpha} e_{\alpha} x\right)\right)+\partial([x, y])+i B(x, y)\right) .
\end{aligned}
$$

Hence, by (2.10) and (2.11),

$$
\begin{aligned}
\hat{L}_{p}(x)= & -\frac{i}{2}\left(\sum_{\alpha, \beta} B\left(e_{\alpha} x e_{\beta}, y\right) \partial\left(\check{e}_{\alpha}\right) \partial\left(\check{e}_{\beta}\right)\right. \\
& \left.+\partial\left(\sum_{\alpha}\left(x e_{\alpha} \check{e}_{\alpha}+\check{e}_{\alpha} e_{\alpha} x\right)-2 \tilde{p} x\right)-i \partial([x, y])+B(x, y)\right) .
\end{aligned}
$$

Let $U$ be a vector space over $\mathbb{C}$ defined as follows:

$$
U=\left\{\begin{array}{ll}
V_{j} \otimes \mathbb{C} & \text { if } \quad \mathbb{D}=\mathbb{R}, \\
V_{j} & \text { if } \quad \mathbb{D}=\mathbb{C} \\
\left.V_{j}\right|_{\mathbb{C}} & \text { if } \quad \mathbb{D}=\mathbb{H} .
\end{array} \text { and } \iota \neq 1,\right.
$$

The complexification $\mathfrak{g}_{\mathbb{C}}$ of $\mathfrak{g}$ may be realized as a Lie subalgebra of $\operatorname{End}(U)$. Specifically, if $\mathbb{D}=\mathbb{R}$ or $\mathbb{H}$, then $\mathfrak{g}_{\mathbb{C}}$ is identified with the Lie algebra of isometries of an appropriate $\mathbb{C}$-bilinear form on $U$ (which might be the zero form). If $\mathbb{D}=\mathbb{C}$ and $\iota \neq 1$ then $\mathfrak{g}_{\mathbb{C}}=\operatorname{End}(U)$. If $\mathbb{D}=\mathbb{C}$ and $\iota=1$ then $\mathfrak{g}_{\mathbb{C}}$ is equal to the direct sum $\mathfrak{g} \oplus \mathfrak{g} \subseteq \operatorname{End}(U)$ via the embedding

$$
\mathfrak{g} \ni y \rightarrow(y, \bar{y}) \in \mathfrak{g} \oplus \mathfrak{g},
$$


where $\mathfrak{g} \ni y \rightarrow \bar{y} \in \mathfrak{g}$ is the complex conjugation with respect to some real form. Let

$$
B_{U}(x, y)=\operatorname{tr}(x y) \quad(x, y \in \operatorname{End}(U))
$$

and let us select

$$
B_{V}(x, y)=\operatorname{tr}_{\mathbb{D} / \mathbb{R}}(x y) \quad\left(x, y \in \operatorname{End}\left(V_{j}\right)\right),
$$

for the form $B$ we have been using so far. Let $u=1$ if $\iota=1$, and let $u=2$ if $\iota \neq 1$. Then a straightforward computation shows that

$$
B_{V}(x, y)=u B_{U}(x, y) \quad(x, y \in \mathfrak{g}) .
$$

Let $f_{\alpha}=e_{\alpha}$ and let $\check{f}_{\alpha}=u \check{e}_{\alpha}, \alpha \in \mathcal{A}$. Then

$$
B_{U}\left(f_{\alpha}, \check{f}_{\beta}\right)=u B_{U}\left(e_{\alpha}, \check{e}_{\beta}\right)=B_{V}\left(e_{\alpha}, \check{e}_{\beta}\right)=\delta_{\alpha, \beta} \quad(\alpha, \beta \in \mathcal{A}) .
$$

Thus $\left\{f_{\alpha} ; \alpha \in \mathcal{A}\right\}$ is a basis of $U$ and $\left\{\check{f}_{\alpha} ; \alpha \in \mathcal{A}\right\}$ is the dual basis with respect to the form $B_{U}$. In terms of these bases, (2.16) may be rewritten as

$$
\begin{aligned}
\hat{L}_{p}(x)= & -\frac{i}{2 u}\left(\sum_{\alpha, \beta} B_{U}\left(f_{\alpha} x f_{\beta}, y\right) \partial\left(\check{f}_{\alpha}\right) \partial\left(\check{f}_{\beta}\right)\right. \\
& \left.+\partial\left(\sum_{\alpha}\left(x f_{\alpha} \check{f}_{\alpha}+\check{f}_{\alpha} f_{\alpha} x\right)-2 u \tilde{p} x\right)-i u \partial([x, y])+u B_{U}(x, y)\right) .
\end{aligned}
$$

Since $\hat{L}_{p}(x)$ is a representation of $\mathfrak{g}_{\mathbb{C}}$, the expression (2.17) does not depend on the particular choice of the basis $\left\{f_{\alpha} ; \alpha \in \mathcal{A}\right\}$.

Let $\mathfrak{g}_{\mathbb{C}, r k \leq k}=\left\{y \in \mathfrak{g}_{\mathbb{C}} ; \operatorname{rank}(y) \leq k\right\}$, where $\operatorname{rank}(y)=\operatorname{dim}_{\mathbb{C}}(y(U))$. As is well known, and easy to check,

$$
\tau_{\mathfrak{g}}\left(\mathfrak{s}_{1, \mathbb{C}}\right)=\left\{\begin{array}{lll}
\mathfrak{g}_{\mathbb{C}, r k \leq d^{\prime}} & \text { if } & \mathbb{D} \neq \mathbb{H} \\
\mathfrak{g}_{\mathbb{C}, r k \leq 2 d^{\prime}} & \text { if } & \mathbb{D}=\mathbb{H} .
\end{array}\right.
$$

Moreover the number $r_{\mathfrak{g}}$ may be expressed in terms of $m=\operatorname{dim}_{\mathbb{C}}(U)$ as follows:

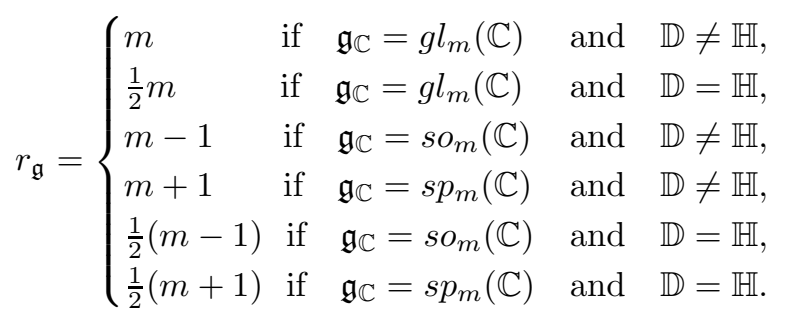

Let

$$
k=\left\{\begin{array}{lll}
d^{\prime} & \text { if } & \mathbb{D} \neq \mathbb{H} \\
2 d^{\prime} & \text { if } & \mathbb{D}=\mathbb{H} .
\end{array}\right.
$$

By inspecting the above formula for $\tau_{\mathfrak{g}}\left(\mathfrak{s}_{1, \mathbb{C}}\right)$ and for $r_{\mathfrak{g}}$, we see that we will be done as soon as we verify the following claim.

Claim 2.18. The operators

(a) $\sum_{\alpha, \beta} B_{U}\left(f_{\alpha} x f_{\beta}, y\right) \partial\left(\check{f}_{\alpha}\right) \partial\left(\check{f}_{\beta}\right)+\partial\left(\sum_{\alpha}\left(x f_{\alpha} \check{f}_{\alpha}+\check{f}_{\alpha} f_{\alpha} x\right)-2 u \tilde{p} x\right) \quad\left(x \in \mathfrak{g}_{\mathbb{C}}\right)$

preserve the ideal

$$
\mathbf{I}\left(\mathfrak{g}_{\mathbb{C}, r k \leq k}\right) \subseteq \mathcal{P}\left(\mathfrak{g}_{\mathbb{C}}\right)
$$


if and only if

$$
2 u \tilde{p}\left\{\begin{array}{lrl}
2 m-k & \text { if } & \mathfrak{g}_{\mathbb{C}}=g l_{m}(\mathbb{C}), \\
m-1-\frac{1}{2} k & \text { if } & \mathfrak{g}_{\mathbb{C}}=s o_{m}(\mathbb{C}), \\
m+1-\frac{1}{2} k & \text { if } & \mathfrak{g}_{\mathbb{C}}=s p_{m}(\mathbb{C}) .
\end{array}\right.
$$

We shall proceed via a case by case analysis.

Case $\mathfrak{g}_{\mathbb{C}}=g l_{m}(\mathbb{C})$. We choose the set $\left\{E_{a, b} ; 1 \leq a, b \leq m\right\}$ to be the basis of $\mathfrak{g}_{\mathbb{C}}$. Here $E_{a, b}$ is the matrix with 1 in the $a^{\text {th }}$ row and $b^{\text {th }}$ column and zeros elsewhere. Then $\check{E}_{a, b}=E_{b, a}$. For $y \in \mathfrak{g}_{\mathbb{C}}$ let $y_{a, b}=\operatorname{tr}\left(y E_{b, a}\right)$. Then a straightforward computation shows that for $x=E_{c, b}$ the operator (2.18.a) coincides with

$$
\sum_{a, d} y_{a, d} \partial\left(E_{a, b}\right) \partial\left(E_{c, d}\right)+(2 m-2 u \tilde{p}) \partial\left(E_{c, b}\right) .
$$

For two subsets $I, J \subseteq\{1,2,3, \ldots, m\}$ of the same cardinality $|I|=|J| \leq m$, let $\operatorname{det}_{I, J}(y)$ denote the determinant of the matrix obtained by deleting all the rows indexed by $\{1,2,3, \ldots, m\} \backslash I$ and all the columns indexed by $\{1,2,3, \ldots, m\} \backslash J$. For $i \in I$ let $i_{I}=|\{1,2,3, \ldots, i\} \cap I|$. This is the position of $i$ in the sequence $I$. If $i$ is the smallest element in $I$, then $i_{I}=1$, and if $i$ is the largest element of $I$, then $i_{I}=|I|$.

The co-factor expansion of $\operatorname{det}_{I, J}(y)$ with respect to the $i^{\text {th }}$ row and the $j^{\text {th }}$ column may be written as

$$
\begin{aligned}
& \operatorname{det}_{I, J}(y)=\sum_{j \in J} y_{i, j}(-1)^{i_{I}+j_{J}} \operatorname{det}_{I \backslash i, J \backslash j}(y) \quad(i \in I), \\
& \operatorname{det}_{I, J}(y)=\sum_{i \in I} y_{i, j}(-1)^{i_{I}+j_{J}} \operatorname{det}_{I \backslash i, J \backslash j}(y) \quad(j \in J) .
\end{aligned}
$$

(Here $I \backslash i=I \backslash\{i\}$, and similarly for $J$.) Hence,

$$
\partial\left(E_{i, j}\right) \operatorname{det}_{I, J}=(-1)^{i_{I}+j_{J}} \operatorname{det}_{I \backslash i, J \backslash j} \quad(i \in I, j \in J) .
$$

Thus for $a, c \in I, a \neq c$, and $b, d \in J, b \neq d$, we have

$$
\partial\left(E_{a, b}\right) \partial\left(E_{c, d}\right) \operatorname{det}_{I, J}=(-1)^{a_{I \backslash c}+b_{J \backslash d}+c_{I}+d_{J}} \operatorname{det}_{I \backslash\{a, c\}, J \backslash\{b, d\}} .
$$

Since

$$
(-1)^{b_{J \backslash d}+d_{J}+d_{J \backslash b}}=-(-1)^{b_{J}},
$$

we see that

$$
\partial\left(E_{a, b}\right) \partial\left(E_{c, d}\right) \operatorname{det}_{I, J}=-(-1)^{b_{J}+c_{I}}(-1)^{a_{I \backslash c}+d_{J \backslash b}} \operatorname{det}_{(I \backslash c) \backslash a,(J \backslash b) \backslash d} .
$$

Therefore, by (2.20),

$$
\sum_{a \in I \backslash c} y_{a, d} \partial\left(E_{a, b}\right) \partial\left(E_{c, d}\right) \operatorname{det}_{I, J}=-(-1)^{b_{J}+c_{I}} \operatorname{det}_{I \backslash c, J \backslash b},
$$

and consequently, for $c \in I$ and $b \in J$,

$$
\sum_{a \in I \backslash c, d \in J \backslash b} y_{a, d} \partial\left(E_{a, b}\right) \partial\left(E_{c, d}\right) \operatorname{det}_{I, J}=-(-1)^{b_{J}+c_{I}}(|I|-1) \operatorname{det}_{I \backslash c, J \backslash b} .
$$

By combining (2.20), (2.21) and (2.24) we see that for arbitrary $1 \leq b, c \leq m$,

$$
\sum_{a, d=1}^{m} y_{a, d} \partial\left(E_{a, b}\right) \partial\left(E_{c, d}\right) \operatorname{det}_{I, J}=-(|I|-1) \partial\left(E_{c, b}\right) \operatorname{det}_{I, J} .
$$


Thus the operator (2.19) applied to $\operatorname{det}_{I, J}$ gives the same result as

$$
(2 m-(|I|-1)-2 u \tilde{p}) \partial\left(E_{c, b}\right) \operatorname{det}_{I, J} .
$$

In particular, the operator (2.19) annihilates all the minors $\operatorname{det}_{I, J}$ with $|I|=k+1$ if and only if

$$
2 m-k-2 u \tilde{p}=0 .
$$

We see from (2.20) and (2.21) that for $b \in J$,

$$
\sum_{a=1}^{m} y_{a, d} \partial\left(E_{a, b}\right) \operatorname{det}_{I, J}=\left\{\begin{array}{lc} 
\pm \operatorname{det}_{I,(J \backslash b) \cup d} & \text { if } d=b \text { or } d \notin J, \\
0 & \text { otherwise, }
\end{array}\right.
$$

and for $c \in I$,

$$
\sum_{d=1}^{m} y_{a, d} \partial\left(E_{c, d}\right) \operatorname{det}_{I, J}= \begin{cases} \pm \operatorname{det}_{(I \backslash c) \cup a, J} & \text { if } a=c \text { or } \quad a \notin I, \\ 0 & \text { otherwise. }\end{cases}
$$

(Here $(I \backslash c) \cup a=(I \backslash\{c\}) \cup\{a\}$, and similarly for $J$.) If $b \notin J$ then the expression (2.28) is zero, and if $c \notin I$ then the expression (2.29) is zero.

Let $\psi \in \mathcal{P}\left(\mathfrak{g}_{\mathbb{C}}\right)$. The operator (2.19) applied to the product $\psi \operatorname{det}_{I, J}$ gives

$$
\begin{aligned}
& \left(\sum_{a, d} y_{a, d} \partial\left(E_{a, b}\right) \partial\left(E_{c, d}\right)+(2 m-2 u \tilde{p}) \partial\left(E_{c, b}\right)\right)(\psi) \operatorname{det}_{I, J} \\
+ & \psi\left(\sum_{a, d} y_{a, d} \partial\left(E_{a, b}\right) \partial\left(E_{c, d}\right)+(2 m-2 u \tilde{p}) \partial\left(E_{c, b}\right)\right)\left(\operatorname{det}_{I, J}\right) \\
+ & \sum_{a}\left(\sum_{d} y_{a, d} \partial\left(E_{c, d}\right) \operatorname{det}_{I, J}\right) \partial\left(E_{a, b}\right) \psi \\
+ & \sum_{d}\left(\sum_{a} y_{a, d} \partial\left(E_{a, b}\right) \operatorname{det}_{I, J}\right) \partial\left(E_{c, d}\right) \psi .
\end{aligned}
$$

As is well known, G-W Theorem 5.2.15], the ideal (2.18.b) is generated by the minors $\operatorname{det}_{I, J}$ with $|I|=|J|=k+1$. Hence the expression (2.30) together with (2.28), (2.29) and (2.26) shows that the operators (2.18.a) preserve this ideal if and only if the condition (2.27) holds. Since this condition is equivalent to the condition (2.18.c), in this case, we are done.

Case $\mathfrak{g}_{\mathbb{C}}=s p_{m}(\mathbb{C})$. Here $m$ is even. Let

$$
\mathcal{J}=\left[\begin{array}{cc}
0 & \mathcal{I} \\
-\mathcal{I} & 0
\end{array}\right], \quad \mathcal{I}=\mathcal{I}_{m / 2}
$$

Let $S M_{m}(\mathbb{C})$ denote the space of symmetric matrices of size $m$, with complex entries. We shall formulate our problem for this space via the following linear isomorphism:

$$
s p_{m}(\mathbb{C}) \ni y \rightarrow y \mathcal{J}^{-1} \in S M_{m}(\mathbb{C})
$$

For $1 \leq a, b, \leq m$ set

$$
\begin{gathered}
S_{a, b}= \begin{cases}\mathcal{J}\left(E_{a, b}+E_{b, a}\right) \mathcal{J} & \text { if } \quad a \neq b, \\
\mathcal{J} E_{a, a} \mathcal{J} & \text { if } \quad a=b,\end{cases} \\
\check{S}_{a, b}=\frac{1}{2}\left(E_{a, b}+E_{b, a}\right), \quad f_{a, b}=\mathcal{J}^{-1} S_{a, b}, \quad \check{f}_{a, b}=\mathcal{J}^{-1} \check{S}_{a, b} .
\end{gathered}
$$


Then the set $\left\{f_{a, b} ; a \leq b\right\}$ is a basis of $s p_{m}(\mathbb{C})$ and the set $\left\{\check{f}_{a, b} ; a \leq b\right\}$ is the dual basis. A straightforward calculation shows that

$$
\sum_{a \leq b} f_{a, b} \check{f}_{a, b}=\sum_{a \leq b} \check{f}_{a, b} f_{a, b}=\frac{m+1}{2} \mathcal{I}_{m} .
$$

For $x, y \in s p_{m}(\mathbb{C})$ set $A=y \mathcal{J}^{-1}$ and $B=x \mathcal{J}^{-1}$. Then

$$
\begin{aligned}
& \sum_{a \leq b} \sum_{e \leq g} \operatorname{tr}\left(y f_{a, b} x f_{e, g}\right) \partial\left(\mathcal{J} \check{f}_{a, b}\right) \partial\left(\mathcal{J} \check{f}_{e, g}\right) \\
= & \sum_{a \leq b} \sum_{e \leq g} \operatorname{tr}\left(A S_{a, b} B S_{e, g}\right) \partial\left(\check{S}_{a, b}\right) \partial\left(\check{S}_{e, g}\right) .
\end{aligned}
$$

By combining (2.32) and (2.33) we see that the isomorphism (2.31) transforms the operator (2.18.a) into

$$
\sum_{a \leq b} \sum_{e \leq g} \operatorname{tr}\left(A S_{a, b} B S_{e, g}\right) \partial\left(\check{S}_{a, b}\right) \partial\left(\check{S}_{e, g}\right)+(m+1-2 u \tilde{p}) \partial(B) .
$$

Let $A_{a, b}=\operatorname{tr}\left(A \check{S}_{a, b}\right)$. A tedious computation shows that for $B=\check{S}_{c, d}, c \leq d$, the operator (2.34) may be written as

$$
\sum_{a, b=1}^{m} A_{a, b} \partial\left(\check{S}_{a, c}\right) \partial\left(\check{S}_{b, d}\right)+(m+1-2 u \tilde{p}) \partial\left(\check{S}_{c, d}\right) .
$$

Let $\mathbf{S I}_{k} \subseteq \mathcal{P}\left(S M_{m}(\mathbb{C})\right)$ denote the image of the ideal (2.18.b) under the map (2.31). As is well known, [G-W, Theorem 5.2.17], this ideal is generated by the minors $\operatorname{det}_{I, J},|I|=|J|=k+1$, restricted to $S M_{m}(\mathbb{C})$. We need to show that the operators (2.35) preserve the ideal $\mathbf{S I}_{k}$ if and only if

$$
m+1-\frac{1}{2} k-2 u \tilde{p}=0 .
$$

As in (2.23) we have

$$
(-1)^{a_{I \backslash c}+c_{I}+c_{I \backslash a}}=-(-1)^{a_{I}} .
$$

Hence, by (2.22),

$$
\partial\left(E_{a, b}\right) \partial\left(E_{c, d}\right) \operatorname{det}_{I, J}=-(-1)^{a_{I}+d_{J}}(-1)^{c_{I \backslash a}+b_{J \backslash d}} \operatorname{det}_{(I \backslash a) \backslash c,(J \backslash d) \backslash b} .
$$

Thus for $A \in g l_{m}(\mathbb{C})$,

$$
\sum_{b \in J \backslash d} A_{c, b} \partial\left(E_{a, b}\right) \partial\left(E_{c, d}\right) \operatorname{det}_{I, J}(A)=-(-1)^{a_{I}+d_{J}} \operatorname{det}_{I \backslash a, J \backslash d}(A),
$$

so that

$$
\sum_{b \in J \backslash d, c \in I \backslash a} A_{c, b} \partial\left(E_{a, b}\right) \partial\left(E_{c, d}\right) \operatorname{det}_{I, J}(A)=-(-1)^{a_{I}+d_{J}}(|I|-1) \operatorname{det}_{I \backslash a, J \backslash d}(A) .
$$

Notice that

$$
(-1)^{a_{I \backslash b}+b_{I}}=-(-1)^{b_{I \backslash a}+a_{I}} \quad(a, b \in I, a \neq b) .
$$

Hence, by (2.22),

$$
\begin{aligned}
& \left(\partial\left(E_{a, c}\right) \partial\left(E_{b, d}\right)+\partial\left(E_{b, c}\right) \partial\left(E_{a, d}\right)\right) \operatorname{det}_{I, J} \\
= & \left((-1)^{a_{I \backslash b}+c_{J \backslash d}+b_{I}+d_{J}}+(-1)^{b_{I \backslash a}+c_{J \backslash d}+a_{I}+d_{J}}\right) \operatorname{det}_{I \backslash\{a, b\}, J \backslash\{c, d\}}=0,
\end{aligned}
$$


and similarly

$$
\left(\partial\left(E_{c, a}\right) \partial\left(E_{d, b}\right)+\partial\left(E_{c, b}\right) \partial\left(E_{d, a}\right)\right) \operatorname{det}_{I, J}=0 .
$$

Let $A \in S M_{m}(\mathbb{C})$. Then (2.25), (2.37), (2.38) and (2.39) imply

$$
\begin{aligned}
& \sum_{a, b=1}^{m} A_{a, b} \partial\left(E_{a, c}+E_{c, a}\right) \partial\left(E_{b, d}+E_{d, b}\right) \operatorname{det}_{I, J} \\
= & \sum_{a, b=1}^{m} A_{a, b} \partial\left(E_{a, c}\right) \partial\left(E_{d, b}\right) \operatorname{det}_{I, J}+\sum_{a, b=1}^{m} A_{a, b} \partial\left(E_{c, a}\right) \partial\left(E_{b, d}\right) \operatorname{det}_{I, J} \\
= & -(-1)^{c_{J}+d_{I}}(|J|-1) \operatorname{det}_{I \backslash d, J \backslash c}-(-1)^{c_{J}+d_{I}}(|J|-1) \operatorname{det}_{I \backslash c, J \backslash d},
\end{aligned}
$$

where $\operatorname{det}_{I \backslash d, J \backslash c}=0$ if $d \notin I$ or $c \notin J$, and similarly for $\operatorname{det}_{I \backslash c, J \backslash d}$. By combining $(2.21)$ and $(2.40)$ we see that

$$
\sum_{a, b=1}^{m} A_{a, b} \partial\left(\check{S}_{a, c}\right) \partial\left(\check{S}_{b, d}\right) \operatorname{det}_{I, J}(A)=-\frac{1}{2}(|I|-1) \partial\left(\check{S}_{c, d}\right) \operatorname{det}_{I, J}(A) .
$$

Hence the operator (2.35) applied to $\operatorname{det}_{I, J}$ coincides with

$$
\left(m+1-\frac{1}{2}(|I|-1)-2 u \tilde{p}\right) \partial\left(\check{S}_{c, d}\right) \operatorname{det}_{I, J} .
$$

Thus the condition (2.36) is necessary for the preservation of the ideal $\mathbf{S I}_{k}$. It remains to check that this condition is also sufficient. But (2.28) and (2.29) imply that for $|I|=|J|=k+1$,

$$
\sum_{a=1}^{m} A_{a, b} \partial\left(\check{S}_{a, c}\right) \operatorname{det}_{I, J}(A) \in \mathbf{S I}_{k} \quad \text { and } \quad \sum_{b=1}^{m} A_{a, b} \partial\left(\check{S}_{b, d}\right) \operatorname{det}_{I, J}(A) \in \mathbf{S I}_{k} .
$$

Hence the sufficiency of the condition (2.36) follows as in the case $\mathfrak{g}_{\mathbb{C}}=g l_{m}(\mathbb{C})$.

Case $\mathfrak{g}_{\mathbb{C}}=s o_{m}(\mathbb{C})$. Let us realize the Lie algebra $s o_{m}(\mathbb{C})$ as the space $A M_{m}(\mathbb{C})$ of the alternating matrices of size $m$. For $1 \leq a, b \leq m$ set

$$
f_{a, b}=-E_{a, b}+E_{b, a}, \quad \check{f}_{a, b}=\frac{1}{2}\left(E_{a, b}-E_{b, a}\right) .
$$

Then the set $\left\{f_{a, b} ; a<b\right\}$ is a basis of $\mathfrak{g}_{\mathbb{C}}$ and the set $\left\{\check{f}_{a, b} ; a<b\right\}$ is the dual basis. A straightforward calculation shows that

$$
\sum_{a<b} f_{a, b} \check{f}_{a, b}=\sum_{a<b} \check{f}_{a, b} f_{a, b}=\frac{m-1}{2} I_{m} .
$$

Hence, the operator (2.18.a) may be written as

$$
\sum_{a<b} \sum_{e<g} \operatorname{tr}\left(y f_{a, b} x f_{e, g}\right) \partial\left(\check{f}_{a, b}\right) \partial\left(\check{f}_{e, g}\right)+(m-1-2 u \tilde{p}) \partial(x) .
$$

Let $y_{a, b}=\operatorname{tr}\left(y E_{b, a}\right)$. Then for $x=\check{S}_{c, d}$ the operator (2.44) is equal to

$$
\sum_{a \neq c} \sum_{b \neq d} y_{a, b} \partial\left(\check{f}_{a, c}\right) \partial\left(\check{f}_{b, d}\right)+(m-1-2 u \tilde{p}) \partial\left(\check{f}_{c, d}\right) .
$$

Since the ideal (2.18.b) is generated by some Pfaffians, we recall a few facts.

Let $n=1,2, \ldots$, and let $I=\{1,2, \ldots, 2 n\}$. For $i \in I$ and $S \subseteq I$ let

$$
i_{S}=|\{1,2, \ldots, i\} \backslash S| \text {. }
$$


For $A \in A M_{2 n}(\mathbb{C})$ and for $i, j \in I(i \neq j)$, let $A^{i, j}$ be the skew-symmetric matrix obtained by removing the columns $i$ and $j$ and the rows $i$ and $j$ from $A$. Let $\operatorname{Pff}(A)$ denote the Pfaffian of $A$, [G-W], (B.2.10)]. Let $A_{i, j}=\operatorname{tr}\left(A \check{f}_{j, i}\right)$, as usual.

Lemma 2.47. For any $i \in I, \operatorname{Pff}(A)=\sum_{i \neq j} A_{i, j}(-1)^{i+j_{i}} \operatorname{Pff}\left(A^{i, j}\right)$.

Proof. By [J p. 336], the formula holds if $i=1$. Let $1<i \leq 2 n$. Let $t$ denote the cyclic permutation

$$
t: 1 \rightarrow 2 \rightarrow 3 \rightarrow \cdots \rightarrow i \rightarrow 1 .
$$

Let $\left\{e_{1}, e_{2}, \ldots, e_{2 n}\right\}$ be the standard basis of $\mathbb{C}^{2 n}$. Define $g \in G L_{2 n}(\mathbb{C})$ by

$$
g\left(e_{l}\right)=e_{t^{-1}(l)} \quad(l \in I) .
$$

Since the lemma holds for $i=1$, we have

$$
\begin{aligned}
& \operatorname{Pff}\left(g^{t} A g\right)=\sum_{j=2}^{2 n} e_{1}^{t}\left(g^{t} A g\right) e_{j}(-1)^{1+j_{1}} \operatorname{Pff}\left(\left(g^{t} A g\right)^{1, j}\right) \\
& =\sum_{j=2}^{2 n} A_{i, g^{-1}(j)}(-1)^{j} \operatorname{Pff}\left(A^{i, g^{-1}(j)}\right) .
\end{aligned}
$$

Since

$$
\operatorname{Pff}\left(g^{t} A g\right)=\operatorname{det}(g) \operatorname{Pf} f(A)=(-1)^{i-1} \operatorname{Pff}(A),
$$

we have

$$
\begin{aligned}
\operatorname{Pff}(A) & =\sum_{j=2}^{2 n} A_{i, g^{-1}(j)}(-1)^{i+j-1} \operatorname{Pff}\left(A^{i, g^{-1}(j)}\right) \\
& =\sum_{l=1}^{i-1} A_{i, l}(-1)^{i+l} \operatorname{Pff}\left(A^{i, l}\right)+\sum_{l=i+1}^{2 n} A_{i, l}(-1)^{i+l-1} \operatorname{Pff}\left(A^{i, l}\right) \\
& =\sum_{j \neq i} A_{i, j}(-1)^{i+j_{i}} \operatorname{Pff}\left(A^{i, j}\right) .
\end{aligned}
$$

Corollary 2.48. For distinct numbers $1 \leq a, b, c, d \leq 2 n$,

$$
\partial\left(\check{f}_{a, b}\right) \operatorname{Pff}(A)=\frac{1}{2}(-1)^{a+b_{a}} \operatorname{Pff}\left(A^{a, b}\right),
$$

$$
\partial\left(\check{f}_{a, b}\right) \partial\left(\check{f}_{c, d}\right) \operatorname{Pff}(A)=\frac{1}{4}(-1)^{a+b_{a}+c_{\{a, b\}}+d_{\{a, b, c\}}} \operatorname{Pff}\left(A^{a, b, c, d}\right),
$$

where $A^{a, b, c, d}=\left(A^{a, b}\right)^{c, d}$.

Corollary 2.49. With the above notation we have

$$
\sum_{a \neq c} A_{a, c} \partial\left(\check{f}_{a, b}\right) \partial\left(\check{f}_{c, d}\right) \operatorname{Pff}(A)=-(n-1) \partial\left(\check{f}_{b, d}\right) \operatorname{Pff}(A) .
$$

Proof. A case by case verification shows that

$$
(-1)^{a+b_{a}}=-(-1)^{b+a_{b}} \quad(1 \leq a, b \leq 2 n, a \neq b) .
$$


By (2.48.a) the left hand side of the equation (2.49) is equal to

$$
\begin{array}{r}
\frac{1}{4} \sum_{a \neq c} \sum_{a \neq b} \sum_{a \neq d} \sum_{c \neq d} A_{a, c}(-1)^{a+b_{a}+c_{\{a, b\}}+d_{\{a, b, c\}}} \operatorname{Pff}\left(A^{a, b, c, d}\right) \\
=\frac{1}{4}(-1)^{b+d_{b}} \sum_{a \neq c} \sum_{a \neq b} \sum_{a \neq d} \sum_{c \neq d}(-1)^{a+b_{a}+c_{\{a, b\}}+d_{\{a, b, c\}}+b+d_{b}+a_{\{b, d\}}+c_{\{a, b, d\}}} \\
\times A_{a, c}(-1)^{a_{\{b, d\}}+c_{\{a, b, d\}}} \operatorname{Pff}\left(A^{a, b, c, d}\right) .
\end{array}
$$

But (2.50) implies that

$$
\begin{aligned}
& (-1)^{a+b_{a}+c_{\{a, b\}}+d_{\{a, b, c\}}+b+d_{b}+a_{\{b, d\}}+c_{\{a, b, d\}}} \\
& =-(-1)^{a_{b}+c_{\{a, b\}}+d_{\{a, b, c\}}+d_{b}+a_{\{b, d\}}+c_{\{a, b, d\}}} \\
& =(-1)^{a_{b}+d_{\{a, b\}}+c_{\{a, b, d\}}+d_{b}+a_{\{b, d\}}+c_{\{a, b, d\}}} \\
& =(-1)^{a_{b}+d_{\{a, b\}}+d_{b}+a_{\{b, d\}}} \\
& =-(-1)^{d_{b}+a_{\{b, d\}}+d_{b}+a_{\{b, c\}}}=-1 .
\end{aligned}
$$

Hence (2.51) is equal to

$$
\begin{aligned}
& -\frac{1}{4}(-1)^{b+d_{b}} \sum_{a \neq b} \sum_{a \neq d} \operatorname{Pff}\left(A^{b, d}\right)=-\frac{1}{4}(2 n-2)(-1)^{b+d_{b}} \operatorname{Pff}\left(A^{b, d}\right) \\
& =-\frac{1}{4}(2 n-2) \partial\left(\check{f}_{b, d}\right) \operatorname{Pff}(A) .
\end{aligned}
$$

Let $I$ denote an increasing sequence $i_{1}<i_{2}<\cdots<i_{2 n}$ of elements $1 \leq i_{l} \leq 2 n$. For $A \in A M_{m}(\mathbb{C})$ define

$$
\operatorname{Pff} f_{I}(A)=\operatorname{Pff}\left(\left(A_{i_{a}, i_{b}}\right)_{1 \leq a, b \leq 2 n}\right) .
$$

This is the Pfaffian of the matrix obtained from $A$ by removing all the columns and rows except those indexed by $I$.

Corollary 2.52. For $1 \leq c, d \leq m$ and $A \in A M_{m}(\mathbb{C})$ we have

$$
\sum_{a \neq c, b \neq d} A_{a, b} \partial\left(\check{f}_{a, c}\right) \partial\left(\check{f}_{b, d}\right) \operatorname{Pf} f_{I}(A)=-\frac{1}{2}(|I|-2) \partial\left(\check{f}_{c, d}\right) P f f_{I}(A) .
$$

Corollary 2.53. The operator (2.45) applied to $\operatorname{Pff}(A)$ is equal to

$$
\left(m-1-\frac{1}{2}(|I|-2)-2 u \tilde{p}\right) \partial\left(\check{f}_{c, d}\right) .
$$

Lemma 2.54. With the above notation we have

(a) $\quad \sum_{a \neq c} A_{a, b} 2 \partial\left(\check{f}_{a, c}\right) \operatorname{Pf} f_{I}(A)= \begin{cases}\operatorname{Pf} f_{(I \backslash c) \cup b}(A) & \text { if } b \notin I \text { and } c \in I, \\ 0 & \text { otherwise, }\end{cases}$

and

(b) $\quad \sum_{b \neq d} A_{a, b} 2 \partial\left(\check{f}_{b, d}\right) \operatorname{Pf} f_{I}(A)= \begin{cases}\operatorname{Pf} f_{(I \backslash d) \cup b}(A) & \text { if } b \notin I \text { and } d \in I, \\ 0 & \text { otherwise, }\end{cases}$

Since the ideal (2.18.b) is generated by the Pfaffians $\operatorname{Pf} f_{I}$, with $|I|=k+2$ (see G-W, Theorem 5.2.18]), the Theorem 2.13 follows from (2.53) and (2.54) via the argument used in the previous cases. 


\section{THE MAPS $\delta_{\mathfrak{g} / \mathfrak{h}}^{\prime}, \delta_{\mathfrak{g} / \mathfrak{z}}^{\prime}$}

Let $\mathfrak{h} \subseteq \mathfrak{g}$ be a Cartan subalgebra, and let $\mathfrak{h}^{r e g}=\left\{x \in \mathfrak{h} ; \operatorname{det}(\operatorname{ad}(x))_{\mathfrak{g} / \mathfrak{h}} \neq 0\right\}$. This is the set of regular elements in $\mathfrak{h}$. Let $\pi_{\mathfrak{g} / \mathfrak{h}}$ denote any analytic square root of the polynomial

$$
\mathfrak{h}^{r e g} \ni x \rightarrow \operatorname{det}(\operatorname{ad}(x))_{\mathfrak{g} / \mathfrak{h}} \in \mathbb{C} .
$$

Recall, $\mathrm{H}-\mathrm{C} 2$, p. 96], the Harish-Chandra radial component map $\delta_{\mathfrak{g} / \mathfrak{h}}^{\prime}$ from the algebra of analytic differential operators on $\mathfrak{g}$ to the algebra of analytic differential operators on $\mathfrak{h}^{\text {reg }}$. For any analytic $A d(G)$-invariant differential operator $D$ on $\mathfrak{g}$, $\delta_{\mathfrak{g} / \mathfrak{h}}^{\prime}(D)$ is the unique analytic differential operator on $\mathfrak{h}^{\text {reg }}$ such that

$$
\left.(D \psi)\right|_{\mathfrak{h}^{\text {reg }}}=\delta_{\mathfrak{g} / \mathfrak{h}}^{\prime}(D)\left(\left.\psi\right|_{\mathfrak{h}^{\text {reg }}}\right) \quad\left(\psi \in C^{\infty}\left(A d(G) \mathfrak{h}^{\text {reg }}\right)^{G}\right)
$$

(see [V] Lemma 13, p. 31]).

The theorem below describes the basic properties of the map $\delta_{\mathfrak{g} / \mathfrak{h}}^{\prime}$, proven by Harish-Chandra, [H-C2, Theorem 1, p. 100], H-C3, Theorem 1, p. 547, Lemma 13, p. 544], Levaseur and Stafford, [L-S3, Theorem 1.1], [L-S2, Theorem 1, p. 365], and Wallach [W, Theorem 2.2].

Theorem 3.2. Let $W$ be the Weyl group for the pair $\left(\mathfrak{g}_{\mathbb{C}}, \mathfrak{h}_{\mathbb{C}}\right)$. For $D \in \mathcal{P} \mathcal{D}(\mathfrak{g})^{G}$ set

$$
\delta_{\mathfrak{g} / \mathfrak{h}}(D)=\pi_{\mathfrak{g} / \mathfrak{h}} \delta_{\mathfrak{g} / \mathfrak{h}}^{\prime}(D) \frac{1}{\pi_{\mathfrak{g} / \mathfrak{h}}}
$$

Then $\delta_{\mathfrak{g} / \mathfrak{h}}(D) \in \mathcal{P} \mathcal{D}(\mathfrak{h})^{W}$ and the map

$$
\delta_{\mathfrak{g} / \mathfrak{h}}: \mathcal{P} \mathcal{D}(\mathfrak{g})^{G} \rightarrow \mathcal{P} \mathcal{D}(\mathfrak{h})^{W}
$$

is a surjective algebra homomorphism.

Let $\alpha: \mathfrak{g}_{\mathbb{C}} \rightarrow \mathcal{P D}\left(\mathfrak{g}_{\mathbb{C}}\right)$ be the derivative of the adjoint action. Thus for $x, y \in \mathfrak{g}$, the local expression of $\alpha(x)$ at $y$ is $[y, x]$. Then

$$
\operatorname{ker}\left(\delta_{\mathfrak{g} / \mathfrak{h}}\right)=\mathcal{P} \mathcal{D}\left(\mathfrak{g}_{\mathbb{C}}\right)^{G} \cap\left(\mathcal{P} \mathcal{D}\left(\mathfrak{g}_{\mathbb{C}}\right) \alpha\left(\mathfrak{g}_{\mathbb{C}}\right)\right) .
$$

Furthermore,

$$
\delta_{\mathfrak{g} / \mathfrak{h}}(q)=\left.q\right|_{\mathfrak{h}}, \quad \delta_{\mathfrak{g} / \mathfrak{h}}(\partial(q))=\partial\left(\left.q\right|_{\mathfrak{h}}\right) \quad\left(q \in \mathcal{S}\left(\mathfrak{g}_{\mathbb{C}}\right)^{G}\right),
$$

and

$$
\delta_{\mathfrak{g} / \mathfrak{h}}(\hat{D})=\delta_{\mathfrak{g} / \mathfrak{h}}(D)^{\wedge} \quad\left(D \in \mathcal{P} \mathcal{D}(\mathfrak{g})^{G}\right) .
$$

Let $\mathfrak{z} \subseteq \mathfrak{g}$ be a reductive Lie subalgebra containing the Cartan subalgebra $\mathfrak{h}$, and let $\mathfrak{z}^{r e g}=\left\{x \in \mathfrak{z} ; \operatorname{det}(\operatorname{ad}(x))_{\mathfrak{g} / \mathfrak{z}} \neq 0\right\}$. This is the set of regular elements in $\mathfrak{z}$. Let $\pi_{\mathfrak{g} / \mathfrak{z}}$ denote any analytic square root of the polynomial

$$
\zeta: \mathfrak{z}^{r e g} \ni x \rightarrow \operatorname{det}(\operatorname{ad}(x))_{\mathfrak{g} / \mathfrak{z}} \in \mathbb{C} .
$$

Recall, [H-C3. p. 545], [H-C5, sec. 7], the Harish-Chandra radial component map $\delta_{\mathfrak{g} / \mathfrak{z}}^{\prime}$ from the algebra of analytic differential operators on $\mathfrak{g}$ to the algebra of analytic differential operators on $\mathfrak{z}^{\text {reg }}$. As shown by Harish-Chandra [H-C3, Lemma 11],

$$
\delta_{\mathfrak{g} / \mathfrak{h}}^{\prime}=\delta_{\mathfrak{z} / \mathfrak{h}}^{\prime} \circ \delta_{\mathfrak{g} / \mathfrak{z}}^{\prime} .
$$


Let $Z \subseteq G$ denote the normalizer $\mathfrak{z}$ in $G$. For any analytic $A d(G)$-invariant differential operator $D$ on $\mathfrak{g}$, the operator $\delta_{\mathfrak{g} / \mathfrak{z}}^{\prime}(D)$ is $A d(Z)$-invariant and the following formula holds:

$$
\left.(D \psi)\right|_{\mathfrak{z}^{\text {reg }}}=\delta_{\mathfrak{g} / \mathfrak{z}}^{\prime}(D)\left(\left.\psi\right|_{\mathfrak{z}^{\text {reg }}}\right) \quad\left(\psi \in C^{\infty}\left(A d(G) \mathfrak{z}^{\text {reg }}\right)^{G}\right) .
$$

Let $\mathcal{P D}(\mathfrak{z})_{\zeta}$ be the algebra of differential operators on $\mathfrak{z}^{\text {reg }}$ generated by $\mathcal{S}(\mathfrak{z} \mathbb{C})$, multiplication by $\zeta^{-1}$, and $\partial(\mathcal{S}(\mathfrak{z} \mathbb{C}))$. Then

$$
\mathcal{P D}(\mathfrak{z})_{\zeta}=\mathbb{C}\left[\zeta^{-1}\right] \mathcal{P} \mathcal{D}(\mathfrak{z})
$$

and since $\zeta$ is $A d(Z)$-invariant,

$$
\mathcal{P} \mathcal{D}(\mathfrak{z})_{\zeta}^{Z}=\mathbb{C}\left[\zeta^{-1}\right] \mathcal{P} \mathcal{D}(\mathfrak{z})^{Z} .
$$

The structure of the map $\delta_{\mathfrak{g} / \mathfrak{z}}^{\prime}$ is much less understood than the structure of the map $\delta_{\mathfrak{g} / \mathfrak{h}}^{\prime}$, if $\mathfrak{z} \neq \mathfrak{h}$. However, by combining [H-C3, Corollary 2, pp. 535, 555 and 556] together with the description of the ideal of the invariant polynomial differential operators on $\mathfrak{z}$ annihilating the invariant distributions, LL-S3, Theorem 1.1], we deduce the following theorem.

Theorem 3.7. For $D \in \mathcal{P} \mathcal{D}(\mathfrak{g})^{G}$ set

$$
\delta_{\mathfrak{g} / \mathfrak{z}}(D)=\pi_{\mathfrak{g} / \mathfrak{z}} \delta_{\mathfrak{g} / \mathfrak{z}}^{\prime}(D) \frac{1}{\pi_{\mathfrak{g} / \mathfrak{z}}} .
$$

Then $\delta_{\mathfrak{g} / \mathfrak{z}}(D) \in \mathcal{P} \mathcal{D}(\mathfrak{z})_{\zeta}^{Z}$. The kernel of the map

$$
\delta_{\mathfrak{z} / \mathfrak{h}}: \mathcal{P} \mathcal{D}(\mathfrak{z})_{\zeta}^{Z} \rightarrow \mathcal{P} \mathcal{D}(\mathfrak{h})_{\zeta}^{W}
$$

is equal to $\mathbf{J}=\mathcal{P} \mathcal{D}(\mathfrak{z})_{\zeta}^{Z} \cap\left(\mathcal{P} \mathcal{D}(\mathfrak{z})_{\zeta} \alpha(\mathfrak{z} \mathbb{C})\right)$. Moreover, the map

$$
\mathcal{P D}(\mathfrak{g})^{G} \ni D \rightarrow \delta_{\mathfrak{g} / \mathfrak{z}}(D)+\mathbf{J} \in \mathcal{P} \mathcal{D}(\mathfrak{z})_{\zeta}^{Z} / \mathbf{J}
$$

is an algebra homomorphism.

\section{THE MAP $\delta_{G / H}^{\prime}$}

Let $\mathcal{U}\left(\mathfrak{g}_{\mathbb{C}}\right)_{d} \subseteq \mathcal{U}\left(\mathfrak{g}_{\mathbb{C}}\right)$ be the subspace spanned by the products of at most $d$ elements of $\mathfrak{g}_{\mathbb{C}}(d=0,1,2, \ldots)$. Then

$$
\mathcal{U}\left(\mathfrak{g}_{\mathbb{C}}\right)_{0} \subseteq \mathcal{U}\left(\mathfrak{g}_{\mathbb{C}}\right)_{1} \subseteq \mathcal{U}\left(\mathfrak{g}_{\mathbb{C}}\right)_{2} \subseteq \cdots
$$

is the standard filtration of the algebra $\mathcal{U}\left(\mathfrak{g}_{\mathbb{C}}\right)$. We shall say that a function

$$
q: G \rightarrow \mathcal{U}\left(\mathfrak{g}_{\mathbb{C}}\right)
$$

is analytic if and only if there is a non-negative integer $d$ such that the range of the function $q$ is contained in $\mathcal{U}_{d}\left(\mathfrak{g}_{\mathbb{C}}\right)$ and the function

$$
q: \mathfrak{g} \rightarrow \mathcal{U}_{d}\left(\mathfrak{g}_{\mathbb{C}}\right)
$$

is analytic. For $q$ as in (4.1) let

$$
L \circ q \Psi(g)=L(q(g)) \Psi(g) \quad\left(\Psi \in C^{\infty}(G), g \in G\right),
$$

where $L$ stands for the left regular representation $(2.2)$. Then $q(g) \in \mathcal{U}\left(\mathfrak{g}_{\mathbb{C}}\right)$ is called the local expression of $L \circ q$ at $g \in G$. For more details see [H-C1 section 4]. 
Let $H \subseteq G$ be the Cartan subgroup with the Lie algebra $\mathfrak{h}$ considered in the previous section. Let $H^{r e g}=\left\{h \in H ; \operatorname{det}(\operatorname{Ad}(h)-1)_{\mathfrak{g} / \mathfrak{h}} \neq 0\right\}$. This is the set of regular elements in $H$. Let $\pi_{G / H}$ denote any analytic square root of the determinant

$$
H^{\text {reg }} \ni h \rightarrow \operatorname{det}\left(\operatorname{Ad}\left(h^{-1}\right)-1\right)_{\mathfrak{g} / \mathfrak{h}} \in \mathbb{C} .
$$

Recall, [H-C1, p. 117], the Harish-Chandra radial component map $\delta_{G / H}^{\prime}$ from the algebra of analytic differential operators on $G$ to the algebra of analytic differential operators on $H^{r e g}$. For any analytic $A d(G)$-invariant differential operator $D$ on $G$, $\delta_{G / H}^{\prime}(D)$ is the unique analytic differential operator on $H^{r e g}$ such that

$$
\left.D \Psi\right|_{H^{r e g}}=\delta_{G / H}^{\prime}(D)\left(\left.\Psi\right|_{H^{r e g}}\right) \quad\left(\Psi \in C^{\infty}\left(A d(G) H^{r e g}\right)^{G}\right)
$$

(see [V, Proposition 6, p. 225]).

Recall the Harish-Chandra isomorphism

$$
\gamma_{\mathfrak{g} / \mathfrak{h}}: \mathcal{U}\left(\mathfrak{g}_{\mathbb{C}}\right)^{G} \rightarrow \mathcal{U}\left(\mathfrak{h}_{\mathbb{C}}\right)^{W}
$$

Then

$$
\delta_{G / H}^{\prime}(L(z))=\frac{1}{\pi_{G / H}} L\left(\gamma_{\mathfrak{g} / \mathfrak{h}}(z)\right) \pi_{G / H} \quad\left(z \in \mathcal{U}\left(\mathfrak{g}_{\mathbb{C}}\right)^{G}\right)
$$

(see [H-C1, Theorem 2, p. 125] and [H-C4 Lemma 13, p. 466]).

\section{The homomorphism $\mathcal{C}$}

In this section we let $B(x, y)=\operatorname{tr}_{\mathbb{D} / \mathbb{R}}(x y), x, y \in \mathfrak{g}$.

Lemma 5.1. For any analytic differential operator $D$ on $\left(-G^{c}\right) \cap A d(G) H^{r e g}$,

$$
\delta_{\mathfrak{g} / \mathfrak{h}}^{\prime}\left(c_{-}^{*}(D)\right)=c_{-}^{*}\left(\delta_{G / H}^{\prime}(D)\right) .
$$

Proof. By (3.1), it will suffice to show that both sides are equal when applied to an arbitrary function $\psi \in C^{\infty}\left(\mathfrak{g}^{c} \cap A d(G) \mathfrak{h}^{\text {reg }}\right)^{G}$. Let $\Psi=\psi \circ c_{-}^{-1}$. Then $\Psi \in C^{\infty}\left(\left(-G^{c}\right) \cap A d(G) H^{r e g}\right)^{G}$ and

$$
\begin{aligned}
\delta_{\mathfrak{g} / \mathfrak{h}}^{\prime}\left(c_{-}^{*}(D)\right)\left(\left.\psi\right|_{\mathfrak{h}^{\text {reg }}}\right) & =\left.\left(c_{-}^{*}(D) \psi\right)\right|_{\mathfrak{h}^{\text {reg }}}=\left.(D \Psi) \circ c_{-}\right|_{\mathfrak{h}^{\text {reg }}} \\
& =\left(\left.(D \Psi)\right|_{H^{\text {reg }}}\right) \circ c_{-} \\
& =\left(\delta_{G / H}^{\prime}(D)\left(\left.\Psi\right|_{H^{\text {reg }}}\right)\right) \circ c_{-} \\
& =\left(\delta_{G / H}^{\prime}(D)\left(\left(\left.\psi\right|_{\mathfrak{h}^{\text {reg }}}\right) \circ c_{-}^{-1}\right)\right) \circ c_{-} \\
& =c_{-}^{*}\left(\delta_{G / H}(D)\right)\left(\left.\psi\right|_{\mathfrak{h}^{\text {reg }}}\right),
\end{aligned}
$$

where the first equality follows from (3.1) and the forth equality from (4.2).

Replacing $\mathfrak{g}$ by $\mathfrak{h}$ in $(2.7)-(2.12)$ yields a representation of $\mathfrak{h}$, denoted $\hat{L}_{\mathfrak{h}, p}$. Thus

$$
\hat{L}_{\mathfrak{h}, p}: \mathcal{U}\left(\mathfrak{h}_{\mathbb{C}}\right) \rightarrow \mathcal{P} \mathcal{D}\left(\mathfrak{h}_{\mathbb{C}}\right)
$$

Let $\epsilon=1$ if $\mathbb{D} \neq \mathbb{H}$, and let $\epsilon=1 / 2$ if $\mathbb{D}=\mathbb{H}$.

Proposition 5.2. For any $p \in \mathbb{C}$,

$$
\delta_{\mathfrak{g} / \mathfrak{h}}\left(\hat{L}_{\mathfrak{g}, p}(z)\right)=\hat{L}_{\mathfrak{h}, p-\left(r_{\mathfrak{g}}-\epsilon\right) / 2}\left(\gamma_{\mathfrak{g} / \mathfrak{h}}(z)\right) \quad\left(z \in \mathcal{U}\left(\mathfrak{g}_{\mathbb{C}}\right)^{G}\right) .
$$


Proof. For $y \in \mathfrak{g}$ let

$$
a_{\mathfrak{g}}(y)= \begin{cases}\operatorname{det}_{\mathbb{R}}(y-1) & \text { if } G \text { is an isometry group, } \\ \operatorname{det}_{\mathbb{R}}(y-1) \operatorname{det}_{\mathbb{R}}(y+1) & \text { if } G \text { is a general linear group. }\end{cases}
$$

Then for $x \in \mathfrak{g}$ and $y \in \mathfrak{g}^{c}$

$$
\left(\frac{1}{a_{\mathfrak{g}}}\left(c_{-}^{*}(L(x)) a_{\mathfrak{g}}\right)(y)= \begin{cases}\frac{1}{2} \operatorname{tr}_{\mathbb{D}} / \mathbb{R}(x y) & \text { if } G \text { is an isometry group, } \\ \operatorname{tr}_{\mathbb{D} / \mathbb{R}}(x y) & \text { if } G \text { is a general linear group. }\end{cases}\right.
$$

Indeed, if $G$ is an isometry group, then

$$
\begin{aligned}
c_{-}^{*}(L(x)) a_{\mathfrak{g}}(y) & =\left.\frac{d}{d t} a_{\mathfrak{g}}\left(y+t \frac{1}{2}(y-1) x(y+1)\right)\right|_{t=0} \\
& =\left.\frac{d}{d t} \operatorname{det}_{\mathbb{R}}\left(y-1+(y-1) \frac{t}{2} x(y+1)\right)\right|_{t=0} \\
& =\left.\operatorname{det}_{\mathbb{R}}(y-1) \frac{d}{d t} \operatorname{det}_{\mathbb{R}}\left(1+\frac{t}{2} x(y+1)\right)\right|_{t=0} \\
& =a_{\mathfrak{g}}(y) \operatorname{tr}_{\mathbb{D} / \mathbb{R}}\left(\frac{1}{2} x(y+1)\right) \\
& =a_{\mathfrak{g}}(y) \frac{1}{2} \operatorname{tr}_{\mathbb{D} / \mathbb{R}}(x y)=a_{\mathfrak{g}}(y) \frac{1}{2} B(x, y) .
\end{aligned}
$$

The computation for a general linear group is analogous. A straightforward argument, as in the proof of Lemma 8, page 544 in [H-C3], shows that for $p \in \mathbb{C}$

$$
\left|a_{\mathfrak{g}}\right|^{-p} c_{-}^{*}(L(x))\left|a_{\mathfrak{g}}\right|^{p}=c_{-}^{*}(L(x))+a_{\mathfrak{g}}^{-1} c_{-}^{*}(L(x)) a_{\mathfrak{g}}
$$

on the set where $a_{\mathfrak{g}}$ is not zero. Hence the definition (2.7) may be rewritten as

$$
L_{\mathfrak{g}, p}(x)=\left|a_{\mathfrak{g}}\right|^{-p} c_{-}^{*}(L(x))\left|a_{\mathfrak{g}}\right|^{p} \quad(x \in \mathfrak{g}) .
$$

Hence, for $z \in \mathcal{U}\left(\mathfrak{g}_{\mathbb{C}}\right)^{G}$,

$$
\begin{aligned}
& \delta_{\mathfrak{g} / \mathfrak{h}}\left(\hat{L}_{\mathfrak{g}, p}(z)\right)=\left(\delta_{\mathfrak{g} / \mathfrak{h}}\left(L_{\mathfrak{g}, p}(z)\right)\right)^{\mathfrak{r}} \\
& =\left(\left|a_{\mathfrak{g}}\right|^{-p} \delta_{\mathfrak{g} / \mathfrak{h}}\left(c_{-}^{*}(L(z))\right)\left|a_{\mathfrak{g}}\right|^{p}\right)^{\mathfrak{r}} \\
& =\left(\left|a_{\mathfrak{g}}\right|^{-p} \pi_{\mathfrak{g} / \mathfrak{h}} \delta_{\mathfrak{g} / \mathfrak{h}}^{\prime}\left(c_{-}^{*}(L(z))\right) \frac{1}{\pi_{\mathfrak{g} / \mathfrak{h}}}\left|a_{\mathfrak{g}}\right|^{p}\right)^{\mathfrak{r}} \\
& =\left(\left|a_{\mathfrak{g}}\right|^{-p} \pi_{\mathfrak{g} / \mathfrak{h}} c_{-}^{*}\left(\delta_{G / H}^{\prime}(L(z)) \frac{1}{\pi_{\mathfrak{g} / \mathfrak{h}}}\left|a_{\mathfrak{g}}\right|^{p}\right)^{\mathfrak{r}}\right. \\
& =\left(\left|a_{\mathfrak{g}}\right|^{-p} \pi_{\mathfrak{g} / \mathfrak{h}} c_{-}^{*}\left(\frac{1}{\pi_{G / H}}\left(L\left(\gamma_{\mathfrak{g} / \mathfrak{h}}(z)\right) \pi_{G / H}\right) \frac{1}{\pi_{\mathfrak{g} / \mathfrak{h}}}\left|a_{\mathfrak{g}}\right|^{p}\right)^{\gamma}\right. \\
& =\left(\left|a_{\mathfrak{g}}\right|^{-p} \frac{\pi_{\mathfrak{g} / \mathfrak{h}}}{c_{-}^{*}\left(\pi_{G / H}\right)} c_{-}^{*}\left(L\left(\gamma_{\mathfrak{g} / \mathfrak{h}}(z)\right) \frac{c_{-}^{*}\left(\pi_{G / H}\right)}{\pi_{\mathfrak{g} / \mathfrak{h}}}\left|a_{\mathfrak{g}}\right|^{p}\right) .\right.
\end{aligned}
$$

A straightforward case by case verification shows that

$$
\frac{\pi_{\mathfrak{g} / \mathfrak{h}}^{2}(y)}{c_{-}^{*}\left(\pi_{G / H}^{2}\right)(y)}=\text { const } \cdot a_{\mathfrak{g}}(y)^{(r-\epsilon)} \quad(y \in \mathfrak{h}) .
$$

Therefore

$$
\delta_{\mathfrak{g} / \mathfrak{h}}\left(\hat{L}_{\mathfrak{g}, p}(z)\right)=\left(\left|a_{\mathfrak{g}}\right|^{-\left(p-\left(r_{\mathfrak{g}}-\epsilon\right) / 2\right)} c_{-}^{*}\left(L\left(\gamma_{\mathfrak{g} / \mathfrak{h}}(z)\right)\left|a_{\mathfrak{g}}\right|^{p-\left(r_{\mathfrak{g}}-\epsilon\right) / 2}\right)^{\mathfrak{r}} \quad\left(z \in \mathcal{U}\left(\mathfrak{g}_{\mathbb{C}}\right)^{G}\right),\right.
$$

which, in view of (5.3), completes the proof. 
Recall the notation (1.15). Notice that any rational differential operator $D$ on $\mathfrak{z}=\mathfrak{h}^{\prime} \oplus \mathfrak{z}^{\prime \prime}$, whose local expression is regular on $\mathfrak{h}^{\prime}$, has a well-defined restriction to a rational differential operator on $\mathfrak{h}^{\prime}$, denoted $\left.D\right|_{\mathfrak{h}^{\prime}}$. (In terms of (2.6), if $D=\partial \circ q$ then $\left.D\right|_{\mathfrak{h}^{\prime}}=\partial \circ\left(\left.q\right|_{\mathfrak{h}^{\prime}}\right)$.)

Fix a Cartan subalgebra $\mathfrak{h}^{\prime \prime} \subseteq \mathfrak{z}^{\prime \prime}$ and let $\mathfrak{h}=\mathfrak{h}^{\prime} \oplus \mathfrak{h}^{\prime \prime}$. Then $\mathfrak{h}$ is a Cartan subalgebra of both $\mathfrak{z}$ and $\mathfrak{g}$. Under the usual identifications

$$
\mathcal{U}(\mathfrak{z} \mathbb{C})^{Z}=\mathcal{U}\left(\mathfrak{h}_{\mathbb{C}}^{\prime}\right)^{W^{\prime}} \otimes \mathcal{U}\left(\mathfrak{z}_{\mathbb{C}}^{\prime \prime}\right)^{Z^{\prime \prime}} \text { and } \mathcal{U}\left(\mathfrak{h}_{\mathbb{C}}^{\prime}\right)^{W^{\prime}} \otimes \mathbb{C}=\mathcal{U}\left(\mathfrak{h}_{\mathbb{C}}^{\prime}\right)^{W^{\prime}}
$$

we define the following algebra homomorphism:

$$
\mathcal{C}: \mathcal{U}\left(\mathfrak{g}_{\mathbb{C}}\right)^{G} \underset{\gamma_{\mathfrak{z} / \mathfrak{h}}^{-1} \circ \gamma_{\mathfrak{g} / \mathfrak{h}}}{\rightarrow} \mathcal{U}(\mathfrak{z} \mathbb{C})^{Z} \underset{1 \otimes \varepsilon_{\mathfrak{z}^{\prime \prime}}}{\rightarrow} \mathcal{U}\left(\mathfrak{h}_{\mathbb{C}}^{\prime}\right)^{W^{\prime}} \underset{\gamma_{\mathfrak{g}^{\prime} / \mathfrak{h}^{\prime}}^{-1}}{\rightarrow} \mathcal{U}\left(\mathfrak{g}_{\mathbb{C}}^{\prime}\right)^{G^{\prime}}
$$

(Here, as in Theorem $2.13, \varepsilon_{\mathfrak{z}^{\prime \prime}}$ is the algebra homomorphism by which $\mathcal{U}\left(\mathfrak{z}^{\prime \prime} \mathbb{C}\right)$ acts on the trivial representation.) The title of this article refers to the map $\mathcal{C}$, which by (4.3) is surjective.

Theorem 5.6. The homomorphism $\mathcal{C}$ is independent of the particular choice of the Cartan subspace $\mathfrak{h}_{1}$ (see (1.14)), or the Cartan subalgebra $\mathfrak{h}^{\prime \prime}$, or the form B used to define the Fourier transform (2.8).

For each $z \in \mathcal{U}\left(\mathfrak{g}_{\mathbb{C}}\right)^{G}$ the differential operator $\delta_{\mathfrak{g} / \mathfrak{z}}^{\prime}\left(\hat{L}_{\mathfrak{g}, r_{\mathfrak{g}}-d^{\prime} / 2}(z)\right)$ is rational on $\mathfrak{z}$, regular on $\mathfrak{h}^{\prime} \subseteq \mathfrak{z}$ and

$$
\left.\delta_{\mathfrak{g} / \mathfrak{z}}^{\prime}\left(\hat{L}_{\mathfrak{g}, r_{\mathfrak{g}}-d^{\prime} / 2}(z)\right)\right|_{\mathfrak{h}^{\prime}}=\delta_{\mathfrak{g}^{\prime} / \mathfrak{h}^{\prime}}^{\prime}\left(\hat{L}_{\mathfrak{g}^{\prime}, r_{\mathfrak{g}^{\prime}}-d / 2}(\mathcal{C}(z))\right) .
$$

Lemma 5.7. For any $t \in \mathbb{C}$ and any $z \in \mathcal{U}\left(\mathfrak{g}_{\mathbb{C}}\right)^{G}$

$$
\delta_{\mathfrak{g} / \mathfrak{z}}\left(\hat{L}_{\mathfrak{g}, t+\left(r_{\mathfrak{g}}-\epsilon\right) / 2}(z)\right)=\hat{L}_{\mathfrak{h}^{\prime}, t} \otimes \hat{L}_{\mathfrak{z}^{\prime \prime}, t+\left(r_{\mathfrak{z}^{\prime \prime}}-\epsilon\right) / 2}\left(\left(\gamma_{\mathfrak{z} / \mathfrak{h}}^{-1} \circ \gamma_{\mathfrak{g} / \mathfrak{h}}\right)(z)\right)+D(z),
$$

where $D(z) \in \mathcal{P} \mathcal{D}(\mathfrak{z})_{\zeta}^{Z} \cap\left(\mathcal{P D}(\mathfrak{z})_{\zeta} \alpha(\mathfrak{z} \mathbb{C})\right)$.

Proof. By Theorem 3.7(b), it will suffice to show that

$$
\delta_{\mathfrak{z} / \mathfrak{h}}\left(\delta_{\mathfrak{g} / \mathfrak{z}}\left(\hat{L}_{\mathfrak{g}, t+\left(r_{\mathfrak{g}}-\epsilon\right) / 2}(z)\right)\right)=\delta_{\mathfrak{z} / \mathfrak{h}}\left(\hat{L}_{\mathfrak{h}^{\prime}, t} \otimes \hat{L}_{\mathfrak{z}^{\prime \prime}, t+\left(r_{\mathfrak{z}^{\prime \prime}}-\epsilon\right) / 2}\left(\left(\gamma_{\mathfrak{z} / \mathfrak{h}}^{-1} \circ \gamma_{\mathfrak{g} / \mathfrak{h}}\right)(z)\right)\right) .
$$

By (3.4) and (5.2) the left hand side of (5.8) is equal to $\hat{L}_{\mathfrak{h}, t}\left(\gamma_{\mathfrak{g} / \mathfrak{h}}(z)\right)$.

As an element of $\mathcal{U}(\mathfrak{z} \mathbb{C})^{Z}=\mathcal{U}\left(\mathfrak{h}_{\mathbb{C}}^{\prime}\right)^{W^{\prime}} \otimes \mathcal{U}\left(\mathfrak{z}_{\mathbb{C}}^{\prime \prime}\right)^{Z^{\prime \prime}},\left(\gamma_{\mathfrak{z} / \mathfrak{h}}^{-1} \circ \gamma_{\mathfrak{g} / \mathfrak{h}}\right)(z)=\sum_{i} z_{i}^{\prime} \otimes z_{i}^{\prime \prime}$, where the sum is finite, $z_{i}^{\prime} \in \mathcal{U}\left(\mathfrak{h}_{\mathbb{C}}^{\prime}\right)^{W^{\prime}}$ and $z_{i}^{\prime \prime} \in \mathcal{U}\left(\mathfrak{z}_{\mathbb{C}}^{\prime \prime}\right)^{Z^{\prime \prime}}$. In these terms the right hand side of (5.8) is equal to

$$
\begin{aligned}
& \sum_{i} \delta_{\mathfrak{z} / \mathfrak{h}}\left(\hat{L}_{\mathfrak{h}^{\prime}, t} \otimes \hat{L}_{\mathfrak{z}^{\prime \prime}, t+\left(r_{\mathfrak{z}^{\prime \prime}}-\epsilon\right) / 2}\left(z_{i}^{\prime} \otimes z_{i}^{\prime \prime}\right)\right) \\
= & \sum_{i} \delta_{\mathfrak{z} / \mathfrak{h}}\left(\hat{L}_{\mathfrak{h}^{\prime}, t}\left(z_{i}^{\prime}\right) \otimes \hat{L}_{\mathfrak{z}^{\prime \prime}, t+\left(r_{\mathfrak{z}^{\prime \prime}}-\epsilon\right) / 2}\left(z_{i}^{\prime \prime}\right)\right) \\
= & \sum_{i} \hat{L}_{\mathfrak{h}^{\prime}, t}\left(z_{i}^{\prime}\right) \otimes \delta_{\mathfrak{z}^{\prime \prime} / \mathfrak{h}^{\prime \prime}}\left(\hat{L}_{\mathfrak{z}^{\prime \prime}, t+\left(r_{\mathfrak{z}^{\prime \prime}}-\epsilon\right) / 2}\left(z_{i}^{\prime \prime}\right)\right) \\
= & \hat{L}_{\mathfrak{h}, t}\left(\sum_{i} z_{i}^{\prime} \otimes \gamma_{\mathfrak{z}^{\prime \prime}} / \mathfrak{h}^{\prime \prime}\right. \\
= & \hat{L}_{\mathfrak{h}, t}\left(\gamma_{\mathfrak{g} / \mathfrak{h}}(z)\right) .
\end{aligned}
$$


Lemma 5.9. Let $t=r_{\mathfrak{g}}-d^{\prime} / 2-\left(r_{\mathfrak{g}}-\epsilon\right) / 2$ and let $t^{\prime}=r_{\mathfrak{g}^{\prime}}-d / 2-\left(r_{\mathfrak{g}^{\prime}}-\epsilon\right) / 2$. Then

$$
\frac{\pi_{\mathfrak{g}^{\prime} / \mathfrak{h}^{\prime}}}{\pi_{\mathfrak{g} / \mathfrak{z}}||_{\mathfrak{h}^{\prime}}} \hat{L}_{\mathfrak{h}^{\prime}, t}(z) \frac{\left.\pi_{\mathfrak{g} / \mathfrak{z}}\right|_{\mathfrak{h}^{\prime}}}{\pi_{\mathfrak{g}^{\prime} / \mathfrak{h}^{\prime}}}=\hat{L}_{\mathfrak{h}^{\prime}, t^{\prime}}(z) \quad\left(z \in \mathcal{U}\left(\mathfrak{h}_{\mathbb{C}}^{\prime}\right)\right)
$$

Proof. There are three cases to consider.

Case $G_{\mathbb{C}}=G L_{d}(\mathbb{C}), G_{\mathbb{C}}^{\prime}=G L_{d^{\prime}}(\mathbb{C})$. Here $t=\left(d-d^{\prime}+1\right) / 2$ and $t^{\prime}=\left(d^{\prime}-d+1\right) / 2$. We may assume that $\mathfrak{h}_{\mathbb{C}}^{\prime}$ is the diagonal Cartan subalgebra of $g l_{d^{\prime}}(\mathbb{C})$. Then the identification (1.14.b) is given by

$$
g l_{d^{\prime}}(\mathbb{C}) \ni \sum_{j=1}^{d^{\prime}} x_{j} E_{j j} \leftrightarrow \sum_{j=1}^{d^{\prime}} x_{j} E_{j j} \in g l_{d}(\mathbb{C}) .
$$

Then we see from definition (2.11) that, with $\partial_{j}=\partial\left(E_{j j}\right)$, we have

$$
\hat{L}_{\mathfrak{h}^{\prime}, t}\left(E_{j j}\right)=-\frac{i}{2}\left(x_{j} \partial_{j}^{2}+2(1-t) \partial_{j}+x_{j}\right) \quad\left(1 \leq j \leq d^{\prime}\right) .
$$

Moreover,

$$
\frac{\pi_{\mathfrak{g}^{\prime} / \mathfrak{h}^{\prime}}\left(\sum_{j=1}^{d^{\prime}} x_{j} E_{j j}\right)}{\pi_{\mathfrak{g} / \mathfrak{z}}\left(\sum_{j=1}^{d^{\prime}} x_{j} E_{j j}\right)}=\left(\prod_{j=1}^{d^{\prime}} x_{j}\right)^{-p} \quad \text { where } \quad p=d-d^{\prime} .
$$

Let $x=x_{j}$ and let $\partial=\partial_{j}$, for short. Then

$$
\begin{aligned}
x^{-p} \partial x^{p} & =\partial+x^{-p}\left[\partial, x^{p}\right]=\partial+p x^{-1} \\
x^{-p} \partial^{2} x^{p} & =\partial^{2}+x^{-p}\left[\partial^{2}, x^{p}\right]=\partial^{2}+x^{-p}\left(\partial\left[\partial, x^{p}\right]+\left[\partial, x^{p}\right] \partial\right) \\
& =\partial^{2}+x^{-p}\left(\left[\partial,\left[\partial, x^{p}\right]\right]+2\left[\partial, x^{p}\right] \partial\right) \\
& =\partial^{2}+x^{-p}\left(p(p-1) x^{p-2}+2 p x^{p-1} \partial\right) \\
& =\partial^{2}+2 p x^{-1} \partial+p(p-1) x^{-2}
\end{aligned}
$$

and therefore

$$
\begin{aligned}
& x^{-p}\left(x \partial^{2}+2(1-t) \partial+x\right) x^{p}=x x^{-p} \partial^{2} x^{p}+2(1-t) x^{-p} \partial x^{p}+x \\
& =x \partial^{2}+2 p \partial+p(p-1) x^{-1}+2(1-t) \partial+2(1-t) p x^{-1}+x \\
& =x \partial^{2}+2(p+1-t) \partial+p(p-1+2(1-t)) x^{-1}+x \\
& =x \partial^{2}+2\left(1-t^{\prime}\right) \partial+x .
\end{aligned}
$$

Hence

$$
\left(\prod_{j=1}^{d^{\prime}} x_{j}\right)^{-p} \hat{L}_{\mathfrak{h}^{\prime}, t}\left(E_{j j}\right)\left(\prod_{j=1}^{d^{\prime}} x_{j}\right)^{p}=\hat{L}_{\mathfrak{h}^{\prime}, t^{\prime}}\left(E_{j j}\right) \quad\left(1 \leq j \leq d^{\prime}\right),
$$

which completes our proof in this case.

Case $G_{\mathbb{C}}=S p_{d}(\mathbb{C}), G_{\mathbb{C}}^{\prime}=O_{d^{\prime}}(\mathbb{C})$. Here $t=\left(d-d^{\prime}+2\right) / 2$ and $t^{\prime}=\left(d^{\prime}-d\right) / 2$. With an appropriate choice of the bilinear form defining the groups $G_{\mathbb{C}}$ and $G^{\prime}$, we may assume that $\mathfrak{h}_{\mathbb{C}}^{\prime}$ is the diagonal Cartan subalgebra of $s o_{d^{\prime}}(\mathbb{C})$ consisting of elements of the form

$$
\sum_{j=1}^{m} x_{j}\left(E_{j j}-E_{d^{\prime}+1-j, d^{\prime}+1-j}\right)
$$


where $m$ is the largest integer less than or equal to $d^{\prime} / 2$, and that the identification (1.14.b) is given by

$$
\text { so }_{d^{\prime}}(\mathbb{C}) \ni \sum_{j=1}^{m} x_{j}\left(E_{j j}-E_{d^{\prime}+1-j, d^{\prime}+1-j}\right) \leftrightarrow \sum_{j=1}^{m} x_{j}\left(E_{j j}-E_{d+1-j, d+1-j}\right) \in s p_{d}(\mathbb{C}) .
$$

Then

$$
\hat{L}_{\mathfrak{h}^{\prime}, t}\left(E_{j j}-E_{d^{\prime}+1-j, d^{\prime}+1-j}\right)=-\frac{i}{2}\left(x_{j} \partial_{j}^{2}+2(1-t) \partial_{j}+x_{j}\right) \quad(1 \leq j \leq m)
$$

and

$$
\frac{\pi_{\mathfrak{g}^{\prime} / \mathfrak{h}^{\prime}}\left(\sum_{j=1}^{m} x_{j}\left(E_{j j}-E_{d^{\prime}+1-j, d^{\prime}+1-j}\right)\right)}{\pi_{\mathfrak{g} / \mathfrak{z}}\left(\sum_{j=1}^{m} x_{j}\left(E_{j j}-E_{d+1-j, d+1-j}\right)\right)}=\left(\prod_{j=1}^{m} x_{j}\right)^{-p} \quad \text { where } \quad p=d-d^{\prime}+1 .
$$

Since $2(1-t)=d^{\prime}-d$, we have

$$
p+1-t=d-d^{\prime}+1+\left(d^{\prime}-d\right) / 2=\left(d-d^{\prime}\right) / 2+1=1-t^{\prime},
$$

and

$$
p-1+2(1-t)=d-d^{\prime}+d^{\prime}-d=0 .
$$

Therefore the computation (5.10) implies the lemma, as in the previous case.

Case $G_{\mathbb{C}}=O_{d}(\mathbb{C}), G_{\mathbb{C}}^{\prime}=S p_{d^{\prime}}(\mathbb{C})(\mathbb{C})$. Here $t=\left(d-d^{\prime}\right) / 2$ and $t^{\prime}=\left(d^{\prime}-d+2\right) / 2$, and with $p=d-d^{\prime}-1$ the above proof adapts to this case.

Proof of Theorem 5.6. Since all the Cartan subspaces $\mathfrak{h}_{1, \mathbb{C}} \subseteq \mathfrak{s}_{1, \mathbb{C}}$ are conjugate under $S_{\mathbb{C}}$, and since all the Harish-Chandra homomorphisms involved in definition (5.5) of the map $\mathcal{C}$ are equivariant with respect to the conjugation by $G_{\mathbb{C}}$, the first claim of Theorem 5.6 is clear.

Let $t \in \mathbb{C}$. Theorem 2.13 implies that we may compose the map

$$
\hat{L}_{\mathfrak{h}^{\prime}, t} \otimes \hat{L}_{\mathfrak{z}^{\prime \prime}, r_{\mathfrak{z}^{\prime \prime}}}: \mathcal{U}\left(h_{\mathbb{C}}^{\prime}\right) \otimes U\left(\mathfrak{z}_{\mathbb{C}}^{\prime \prime}\right) \rightarrow \mathcal{P} \mathcal{D}\left(\mathfrak{h}^{\prime}\right) \otimes \mathcal{P} \mathcal{D}\left(\mathfrak{z}^{\prime \prime}\right)=\mathcal{P} \mathcal{D}\left(\mathfrak{h}^{\prime} \oplus \mathfrak{z}^{\prime \prime}\right)
$$

with the restriction from $\mathfrak{h}^{\prime} \oplus \mathfrak{z}^{\prime \prime}$ to $\mathfrak{h}^{\prime}$, and obtain

$$
\left.\left(\hat{L}_{\mathfrak{h}^{\prime}, t} \otimes \hat{L}_{\mathfrak{z}^{\prime \prime}, r_{\mathfrak{z}^{\prime \prime}}}\right)\right|_{\mathfrak{h}^{\prime}}=\hat{L}_{\mathfrak{h}^{\prime}, t} \otimes \varepsilon_{\mathfrak{z}^{\prime \prime}}: \mathcal{U}\left(h_{\mathbb{C}}^{\prime}\right) \otimes U\left(\mathfrak{z}_{\mathbb{C}}^{\prime \prime}\right) \rightarrow \mathcal{P} \mathcal{D}\left(\mathfrak{h}^{\prime}\right) \otimes \mathbb{C}=\mathcal{P} \mathcal{D}\left(\mathfrak{h}^{\prime}\right) .
$$

Furthermore

$$
\frac{1}{\pi_{\mathfrak{g} / \mathfrak{z}}} \hat{L}_{\mathfrak{h}^{\prime}, t} \otimes \hat{L}_{\mathfrak{z}^{\prime \prime}, r_{\mathfrak{z}^{\prime \prime}}} \pi_{\mathfrak{g} / \mathfrak{z}}=\frac{1}{\pi_{\mathfrak{g}^{\prime}} / \mathfrak{h}^{\prime}} \frac{\pi_{\mathfrak{g}^{\prime} / \mathfrak{h}^{\prime}}}{\pi_{\mathfrak{g} / \mathfrak{z}}} \hat{L}_{\mathfrak{h}^{\prime}, t} \otimes \hat{L}_{\mathfrak{z}^{\prime \prime}, r_{\mathfrak{z}^{\prime}} \prime} \frac{\pi_{\mathfrak{g} / \mathfrak{z}}}{\pi_{\mathfrak{g}^{\prime} / \mathfrak{h}^{\prime}}} \pi_{\mathfrak{g}^{\prime} / \mathfrak{h}^{\prime}}
$$

By restricting to $\mathfrak{h}^{\prime}$ we get

$$
\frac{1}{\pi_{\mathfrak{g}^{\prime} / \mathfrak{h}^{\prime}}} \frac{\pi_{\mathfrak{g}^{\prime}} / \mathfrak{h}^{\prime}}{\left.\pi_{\mathfrak{g} / \mathfrak{z}}\right|_{\mathfrak{h}^{\prime}}} \hat{L}_{\mathfrak{h}^{\prime}, t} \frac{\left.\pi_{\mathfrak{g} / \mathfrak{z}}\right|_{\mathfrak{h}^{\prime}}}{\pi_{\mathfrak{g}^{\prime} / \mathfrak{h}^{\prime}}} \otimes \varepsilon_{\mathfrak{z}^{\prime \prime}} \pi_{\mathfrak{g}^{\prime} / \mathfrak{h}^{\prime}} .
$$

Let $t$ be such that $t+\left(r_{\mathfrak{g}}-\epsilon\right) / 2=r_{\mathfrak{g}}-d^{\prime} / 2$ and let $t^{\prime}=r_{\mathfrak{g}^{\prime}}-d^{\prime} / 2-\left(r_{\mathfrak{g}^{\prime}}-\epsilon\right) / 2$. Then, by Lemma 5.9, the expression (5.11) simplifies to

$$
\frac{1}{\pi_{\mathfrak{g}^{\prime} / \mathfrak{h}^{\prime}}} \hat{L}_{\mathfrak{h}^{\prime}, t^{\prime}} \otimes \varepsilon_{\mathfrak{z}^{\prime \prime}} \pi_{\mathfrak{g}^{\prime} / \mathfrak{h}^{\prime}} .
$$

The following formula is easy to check:

$$
r_{\mathfrak{g}}-r_{\mathfrak{z}^{\prime \prime}}=d^{\prime} .
$$


Hence, $t+\left(r_{\mathfrak{z}^{\prime \prime}}-\epsilon\right) / 2=r_{\mathfrak{z}^{\prime \prime}}$. Let $z \in \mathcal{U}\left(\mathfrak{g}_{\mathbb{C}}\right)^{G}$. Then, in terms of Lemma 5.7, $\left.D(z)\right|_{\mathfrak{h}^{\prime}}=0$. Therefore

$$
\begin{aligned}
& \left.\delta_{\mathfrak{g} / \mathfrak{z}}^{\prime}\left(\hat{L}_{\mathfrak{g}, r_{\mathfrak{g}}-d^{\prime} / 2}(z)\right)\right|_{\mathfrak{h}} \\
& =\frac{1}{\pi_{\mathfrak{g}^{\prime} / \mathfrak{h}^{\prime}}}\left(\hat{L}_{\mathfrak{h}^{\prime}, t^{\prime}} \otimes \varepsilon_{\mathfrak{z}^{\prime \prime}}\right) \circ\left(\gamma_{\mathfrak{z} / \mathfrak{h}}^{-1} \circ \gamma_{\mathfrak{g} / \mathfrak{h}}\right)(z) \pi_{\mathfrak{g}^{\prime} / \mathfrak{h}^{\prime}} \quad \text { by }(5.7) \\
& =\frac{1}{\pi_{\mathfrak{g}^{\prime} / \mathfrak{h}^{\prime}}} \hat{L}_{\mathfrak{h}^{\prime}, t^{\prime}}\left(\left(1 \otimes \varepsilon_{\mathfrak{z}^{\prime \prime}}\right) \circ\left(\gamma_{\mathfrak{z} / \mathfrak{h}}^{-1} \circ \gamma_{\mathfrak{g} / \mathfrak{h}}\right)(z)\right) \pi_{\mathfrak{g}^{\prime} / \mathfrak{h}^{\prime}} \\
& =\frac{1}{\pi_{\mathfrak{g}^{\prime} / \mathfrak{h}^{\prime}}} \hat{L}_{\mathfrak{h}^{\prime}, t^{\prime}}\left(\gamma_{\mathfrak{g}^{\prime} / \mathfrak{h}^{\prime}}(\mathcal{C}(z))\right) \pi_{\mathfrak{g}^{\prime} / \mathfrak{h}^{\prime}} \quad \text { by }(5.5) \\
& =\delta_{\mathfrak{g}^{\prime} / \mathfrak{h}^{\prime}}^{\prime}\left(\hat{L}_{\mathfrak{g}^{\prime}, r_{\mathfrak{g}^{\prime}}-d / 2}(\mathcal{C}(z))\right) \quad \text { by }(5.2) \text {. }
\end{aligned}
$$

\section{The OSCILlator RePRESENTATION}

Recall the symplectic space $\left(\mathfrak{s}_{1},\langle\rangle,\right),(1.10)$. Let $S p$ and $s p \subseteq \operatorname{End}\left(\mathfrak{s}_{1}\right)$ denote the symplectic group and the symplectic Lie algebra, respectively. Set

$$
\tilde{S p}^{c}=\left\{(g, \xi) \in S p \times \mathbb{C}^{\times}, \xi^{2}=\operatorname{det}(i(g-1))^{-1}\right\} .
$$

This is a real analytic manifold and a two-fold covering of $S p^{c}$ via the map

$$
\tilde{S p}{ }^{c} \ni \tilde{g}=(g, \xi) \rightarrow g \in S p^{c} .
$$

Let $\operatorname{dim}\left(\mathfrak{s}_{1}\right)=2 n$ and let

$$
\operatorname{chc}(x)=2^{n}|\operatorname{det}(x)|^{-1 / 2} \exp \left(i \frac{\pi}{4} \operatorname{sgn}\langle x,\rangle\right) \quad(x \in \operatorname{sp}, \operatorname{det}(x) \neq 0),
$$

where "sgn" stands for the signature of the symmetric bilinear form $\langle x$,$\rangle , which is$ the difference between the dimension of the maximal subspace on which the form is positive definite, and the dimension of the maximal subspace on which the form is negative definite.

For $\left(g_{1}, \xi_{1}\right),\left(g_{2}, \xi_{2}\right) \in S p^{c}$ with $c\left(g_{1}\right)+c\left(g_{2}\right)$ invertible in $\operatorname{End}\left(\mathfrak{s}_{1}\right)$ let

$$
\left(g_{1}, \xi_{1}\right) \cdot\left(g_{2}, \xi_{2}\right)=\left(g_{1} g_{2}, \xi_{1} \xi_{2} \operatorname{chc}\left(c\left(g_{1}\right)+c\left(g_{2}\right)\right)\right) .
$$

Theorem 6.4 (Howe, [H2, section 17]). Up to a group isomorphism, there is a unique connected Lie group $\tilde{S p}$ containing $\tilde{S p}^{c}$, with multiplication given by (6.3) on the indicated subset of $\tilde{S p}^{c} \times \tilde{S p}^{c}$, and such that the map (6.1) extends to a double covering homomorphism

$$
\tilde{S p} \ni \tilde{g} \rightarrow g \in S p .
$$

Let

$$
\chi_{x}(w)=\exp \left(2 \pi i \frac{1}{4}\langle x w, w\rangle\right) \quad\left(x \in s p, w \in \mathfrak{s}_{1}\right) .
$$

For $f, f^{\prime} \in S\left(\mathfrak{s}_{1}\right)$, the Schwartz space of $\mathfrak{s}_{1}$, we have the twisted convolution, $\mathrm{H} 2$, section 18],

$$
f^{\prime} \natural f\left(w^{\prime}\right)=\int_{\mathfrak{s}_{1}} f^{\prime}(w) f\left(w^{\prime}-w\right) \chi\left(\frac{1}{2}\left\langle w, w^{\prime}\right\rangle\right) d w \quad\left(w^{\prime} \in \mathfrak{s}_{1}\right) .
$$


As is easy to check, $f^{\prime} \natural f \in S\left(\mathfrak{s}_{1}\right)$. For $f \in S\left(\mathfrak{s}_{1}\right)$ and $y \in s p$, with $1-y$ invertible, the formula

$$
\int_{\mathfrak{s}_{1}} \chi_{y}(w) f\left(w^{\prime}-w\right) \chi\left(\frac{1}{2}\left\langle w, w^{\prime}\right\rangle\right) d w=\chi_{y}\left(w^{\prime}\right) \int_{\mathfrak{s}_{1}} \chi\left(\frac{1}{2}\left\langle(1-y) w^{\prime}, w\right\rangle\right) \chi_{y}(w) f(w) d w
$$

defines a Schwartz function of $w^{\prime} \in \mathfrak{s}_{1}$. By analogy with (6.5) we denote it by $\chi_{y} \llbracket f$. Suppose $x \in s p^{c}$. Then, by the same argument, $\chi_{x} \natural\left(\chi_{y} \natural f\right) \in S\left(\mathfrak{s}_{1}\right)$. Suppose $x+y$ is invertible. Let $z=(y-1)(x+y)^{-1}(x-1)+1$. Then $z \in s p^{c}$ and

$$
\chi_{x} \natural\left(\chi_{y} \natural f\right)=\operatorname{chc}(x+y) \chi_{z} \natural f
$$

(see [H2, (16.2.2a)]). Thus

$$
\begin{aligned}
& \chi_{x} \downarrow \chi_{y}=\operatorname{chc}(x+y) \chi_{z} \\
& \quad\left(x, y \in s p^{c}, \operatorname{det}(x+y) \neq 0, z=(y-1)(x+y)^{-1}(x-1)+1\right) .
\end{aligned}
$$

Define the following functions:

$$
\begin{aligned}
& \Theta: \tilde{S p}^{c} \ni \tilde{g}=(g, \xi) \rightarrow \xi \in \mathbb{C}, \\
& T: \tilde{S p}^{c} \ni \tilde{g}=(g, \xi) \rightarrow \Theta(\tilde{g}) \chi_{c(g)} d w \in S^{*}\left(\mathfrak{s}_{1}\right) .
\end{aligned}
$$

(Then $\Theta$ is the character of the oscillator representation we are considering here.)

Theorem 6.8 ([H2]). The map $T$ extends to a unique injective continuous map $T: \tilde{S p} \rightarrow S^{*}\left(\mathfrak{s}_{1}\right)$ and the following formulas hold:

$$
\begin{aligned}
& T\left(\tilde{g}_{1}\right) \downarrow T\left(\tilde{g}_{2}\right)=T\left(\tilde{g}_{1} \cdot \tilde{g}_{2}\right) \quad\left(\tilde{g}_{1}, \tilde{g}_{2} \in \tilde{S p}^{c}, \operatorname{det}\left(c\left(g_{1}\right)+c\left(g_{2}\right)\right) \neq 0\right), \\
& T(1)=\delta, \text { the Dirac delta at the origin. }
\end{aligned}
$$

Furthermore, the formulas

$$
\begin{aligned}
T(x) \natural \phi & =\left.\frac{d}{d t} T(\exp (t x)) \natural \phi\right|_{t=0} & & \left(x \in s p, \phi \in S\left(\mathfrak{s}_{1}\right)\right), \\
\phi \curvearrowleft T(x) & =\left.\frac{d}{d t} \phi \llbracket T(\exp (t x))\right|_{t=0} & & \left(x \in s p, \phi \in S\left(\mathfrak{s}_{1}\right)\right)
\end{aligned}
$$

define representations of the Lie algebra sp on $S\left(\mathfrak{s}_{1}\right)$ via polynomial differential operators $T(x) \natural$ and $\sharp T(x) \in \mathcal{P} \mathcal{D}\left(\mathfrak{s}_{1}\right)$. Specifically, if $\left\{e_{1}, e_{2}, \ldots, e_{n}, f_{1}, f_{2}, \ldots, f_{n}\right\}$ is a basis of $\mathfrak{s}_{1}$ such that

$$
\left\langle e_{j}, f_{k}\right\rangle=\delta_{j, k}, \quad\left\langle e_{j}, e_{k}\right\rangle=0, \quad\left\langle f_{j}, f_{k}\right\rangle=0 \quad(1 \leq j, k \leq n),
$$

then for $x \in s p, \phi \in S\left(\mathfrak{s}_{1}\right)$ and $w \in \mathfrak{s}_{1}$,

(c)

$$
\begin{aligned}
T(x) \natural \phi(w) & =\partial\left(\frac{1}{4 \pi i} \sum_{j, k=1}^{n}\left(2\left\langle f_{k}, x e_{j}\right\rangle e_{k} f_{j}+\left\langle x e_{j}, e_{k}\right\rangle f_{k} f_{j}+\left\langle x f_{j}, f_{k}\right\rangle e_{k} e_{j}\right)\right. \\
& \left.+\frac{1}{2} \sum_{j=1}^{n}\left(\left\langle f_{j}, x w\right\rangle e_{j}+\left\langle x w, e_{j}\right\rangle f_{j}\right)\right) \phi(w)+\frac{\pi i}{4}\langle x w, w\rangle \phi(w), \\
\phi \llbracket T(x)(w)= & \partial\left(\frac{1}{4 \pi i} \sum_{j, k=1}^{n}\left(2\left\langle f_{k}, x e_{j}\right\rangle e_{k} f_{j}+\left\langle x e_{j}, e_{k}\right\rangle f_{k} f_{j}+\left\langle x f_{j}, f_{k}\right\rangle e_{k} e_{j}\right)\right. \\
& \left.-\frac{1}{2} \sum_{j=1}^{n}\left(\left\langle f_{j}, x w\right\rangle e_{j}+\left\langle x w, e_{j}\right\rangle f_{j}\right)\right) \phi(w)+\frac{\pi i}{4}\langle x w, w\rangle \phi(w) .
\end{aligned}
$$


Recall the representation $\hat{L}_{\mathfrak{g}, p},(2.11)$. Choose the bilinear form $B$, used to define the Fourier transform (2.8), to be

$$
B(x, y)= \begin{cases}-(-1)^{j} 2 \operatorname{tr}_{\mathbb{D} / \mathbb{R}}(x y) & \text { if } \quad x, y \in \mathfrak{g}, \\ -(-1)^{i} 2 \operatorname{tr}_{\mathbb{D} / \mathbb{R}}(x y) & \text { if } \quad x, y \in \mathfrak{g}^{\prime}\end{cases}
$$

Theorem 6.10. With the notation (1.16) we have,

$$
\begin{aligned}
& T(x) \mathfrak{\natural}\left(\psi \circ \tau_{\mathfrak{g}}\right)=\left(\hat{L}_{\mathfrak{g}, r_{\mathfrak{g}}-d^{\prime} / 2}(x) \psi\right) \circ \tau_{\mathfrak{g}} \quad\left(x \in \mathfrak{g}, \quad \psi \in C^{\infty}(\mathfrak{g})\right), \\
& T\left(x^{\prime}\right) \mathfrak{\natural}\left(\psi^{\prime} \circ \tau_{\mathfrak{g}^{\prime}}\right)=\left(\hat{L}_{\mathfrak{g}^{\prime}, r_{\mathfrak{g}^{\prime}}-d / 2}\left(x^{\prime}\right) \psi^{\prime}\right) \circ \tau_{\mathfrak{g}^{\prime}} \quad\left(x^{\prime} \in \mathfrak{g}^{\prime}, \psi^{\prime} \in C^{\infty}\left(\mathfrak{g}^{\prime}\right)\right) .
\end{aligned}
$$

Corollary 6.11. Let $\psi \in C^{\infty}(\mathfrak{g})$ and $\psi^{\prime} \in C^{\infty}\left(\mathfrak{g}^{\prime}\right)$ be such that $\psi \circ \tau_{\mathfrak{g}}=\psi^{\prime} \circ \tau_{\mathfrak{g}^{\prime}}$. Then for $z \in \mathcal{U}\left(\mathfrak{g}_{\mathbb{C}}\right)^{G}$,

$$
\begin{aligned}
T(z) \mathfrak{\natural}\left(\psi \circ \tau_{\mathfrak{g}}\right) & =\left(\hat{L}_{\mathfrak{g}, r_{\mathfrak{g}}-d^{\prime} / 2}(z) \psi\right) \circ \tau_{\mathfrak{g}} \\
& =\left(\hat{L}_{\mathfrak{g}^{\prime}, r_{\mathfrak{g}^{\prime}}-d / 2}(\mathcal{C}(z)) \psi^{\prime}\right) \circ \tau_{\mathfrak{g}^{\prime}} \\
& =T(\mathcal{C}(z)) \downarrow\left(\psi^{\prime} \circ \tau_{\mathfrak{g}^{\prime}}\right) .
\end{aligned}
$$

We have to admit at this point that our methods, designed to work for invariant distributions, are not sufficient to show the following equality of differential operators on $\mathfrak{s}_{1}$ :

$$
T(z) \downarrow=T(\mathcal{C}(z)) \emptyset \quad\left(z \in \mathcal{U}\left(\mathfrak{g}_{\mathbb{C}}\right)^{G}\right) .
$$

(The equality (6.12) is more general than the equality of Corollary 6.11 , because it can be tested on an arbitrary smooth function defined on $\mathfrak{s}_{1}$, rather than a function of the form $\psi \circ \tau_{\mathfrak{g}}=\psi^{\prime} \circ \tau_{\mathfrak{g}^{\prime}}$.) However, we know from the theory of the oscillator representation (see [H0, Theorem 7], [P2] (1.7)]) that there is a homomorphism

$$
\mathcal{C}^{\prime}: \mathcal{U}\left(\mathfrak{g}_{\mathbb{C}}\right)^{G} \rightarrow \mathcal{U}\left(\mathfrak{g}^{\prime} \mathbb{C}\right)^{G^{\prime}}
$$

such that the equation $(6.12)$ holds with $\mathcal{C}$ replaced by $\mathcal{C}^{\prime}$. Let $\psi$ and $\psi^{\prime}$ be as in (6.11). Then, by $(6.10)$,

$$
\begin{aligned}
& \left(\hat{L}_{\mathfrak{g}^{\prime}, r_{\mathfrak{g}^{\prime}}-d / 2}(\mathcal{C}(z)) \psi^{\prime}\right) \circ \tau_{\mathfrak{g}^{\prime}}=T(\mathcal{C}(z)) \downarrow\left(\psi^{\prime} \circ \tau_{\mathfrak{g}^{\prime}}\right) \\
& =T(z) \downarrow\left(\psi \circ \tau_{\mathfrak{g}}\right)=T\left(\mathcal{C}^{\prime}(z)\right) \mathfrak{\natural}\left(\psi \circ \tau_{\mathfrak{g}}\right) \\
& =T\left(\mathcal{C}^{\prime}(z)\right) \downarrow\left(\psi^{\prime} \circ \tau_{\mathfrak{g}^{\prime}}\right)=\left(\hat{L}_{\mathfrak{g}^{\prime}, r_{\mathfrak{g}^{\prime}}-d / 2}\left(\mathcal{C}^{\prime}(z)\right) \psi^{\prime}\right) \circ \tau_{\mathfrak{g}^{\prime}} .
\end{aligned}
$$

Thus for all $\psi^{\prime} \in C^{\infty}\left(\mathfrak{g}^{\prime}\right)^{G^{\prime}}$, supported on the set of regular semisimple elements,

$$
\left(\hat{L}_{\mathfrak{g}^{\prime}, r_{\mathfrak{g}^{\prime}}-d / 2}\left(\mathcal{C}^{\prime}(z)\right) \psi^{\prime}\right) \circ \tau_{\mathfrak{g}^{\prime}}=\left(\hat{L}_{\mathfrak{g}^{\prime}, r_{\mathfrak{g}^{\prime}}-d / 2}(\mathcal{C}(z)) \psi^{\prime}\right) \circ \tau_{\mathfrak{g}^{\prime}} \quad\left(z \in \mathcal{U}\left(\mathfrak{g}_{\mathbb{C}}\right)^{G}\right) .
$$

The image of $\mathfrak{s}_{1}$ under the map $\tau_{\mathfrak{g}^{\prime}}$ has a non-empty interior. Since, by (5.3), the maps

$$
\hat{L}_{\mathfrak{g}^{\prime}, p}: \mathcal{U}\left(\mathfrak{g}_{\mathbb{C}}^{\prime}\right) \rightarrow \mathcal{P} \mathcal{D}\left(\mathfrak{g}^{\prime}\right)
$$

are injective for all $p \in \mathbb{C}$, and since a polynomial differential operator is determined by its restriction to any non-empty open set, we have $\mathcal{C}^{\prime}(z)=\mathcal{C}(z)$ for all $z \in$ $\mathcal{U}\left(\mathfrak{g}_{\mathbb{C}}\right)^{G}$, and therefore the equation (6.12) holds.

Thus the homomorphism $\mathcal{C}$, constructed using the methods of Harish-Chandra, coincides with the homomorphism $\mathcal{C}^{\prime}$, which for a dual pair of general linear groups is determined by the Capelli identities. In order to complete these remarks we mention that for a dual pair of general linear groups the kernel of the map

$$
\mathcal{U}\left(\mathfrak{g}_{\mathbb{C}}\right) \ni z \rightarrow T(z) \natural \in \mathcal{P} \mathcal{D}\left(\mathfrak{s}_{1}\right)
$$


has been determined recently, in terms of generators, by Victor Protsak $[\mathrm{Pr}$ Theorem 3.7.1].

Proof of Theorem 6.10. We consider the group $G$. The proof for $G^{\prime}$ is identical. A straightforward computation shows that one may normalize the Haar measure $d g$ on $G$ and the Lebesgue measure $d x$ on $\mathfrak{g}$ so that

$$
\int_{G} \Phi(g) d g=\int_{\mathfrak{g}} \Phi \circ c(x)\left|a_{\mathfrak{g}}(x)\right|^{-r_{\mathfrak{g}}} d x=\int_{\mathfrak{g}} \Phi \circ c_{-}(x)\left|a_{\mathfrak{g}}(x)\right|^{-r_{\mathfrak{g}}} d x,
$$

for all suitable test functions $\Phi$. Also, it is easy to check from definition (6.7) that for any lift $\tilde{c}: s p^{c}: \rightarrow \tilde{S p}^{c}$ of the Cayley transform $c: s p^{c}: \rightarrow S p^{c}$,

$$
\Theta(\tilde{c}(x))^{2}=(-4)^{-n} a_{\mathfrak{g}}(x)^{d^{\prime}} \quad\left(x \in \mathfrak{g}^{c}\right) .
$$

Hence there is a real analytic lift $\tilde{c}$ such that

$$
\Theta(\tilde{c}(x))=(2 i)^{-n}\left|a_{\mathfrak{g}}(x)\right|^{d^{\prime} / 2} \quad\left(x \in \mathfrak{g}^{c}\right) .
$$

As explained in section 1 , we may view $G$ as a subgroup of $S p$. Let $\tilde{G} \subseteq \tilde{S p}$ be the preimage of $G$ under the covering map (6.4). Set

$$
\tilde{c}_{-}(x)=\tilde{c}(x) \tilde{c}(0)^{-1} \quad\left(x \in \mathfrak{g}^{c}\right) .
$$

Then, in particular, $\tilde{c}_{-}(0)$ is the identity of the group $\tilde{G}$. Let $d \tilde{g}$ be the Haar measure on $\tilde{G}$ so that formula (6.13) holds with $G$ replaced by $\tilde{G}$ and $c$ replaced by $\tilde{c}$. Let $x \in \mathfrak{g}$ and $\Phi \in C_{c}^{\infty}(\tilde{G})$. We see from Theorem 6.8 that the following computation is valid:

$$
\begin{aligned}
& T(x) \natural \int_{\tilde{G}} \Phi(\tilde{g}) T(\tilde{g}) d \tilde{g} \\
& =\left.\frac{1}{2} \frac{d}{d t} T\left(\tilde{c}_{-}(t x)\right) \natural \int_{\tilde{G}} \Phi(\tilde{g}) T(\tilde{g}) d \tilde{g}\right|_{t=0} \\
& =\left.\frac{1}{2} \frac{d}{d t} \int_{\tilde{G}} \Phi(\tilde{g}) T\left(\tilde{c}_{-}(t x) \tilde{g}\right) d \tilde{g}\right|_{t=0} \\
& =\left.\int_{\tilde{G}} \frac{1}{2} \frac{d}{d t} \Phi\left(\tilde{c}_{-}(t x)^{-1} \tilde{g}\right) T(\tilde{g}) d \tilde{g}\right|_{t=0} \\
& =\int_{\tilde{G}}(L(x) \Phi(\tilde{g})) T(\tilde{g}) d \tilde{g} .
\end{aligned}
$$

Thus

$$
T(x) \natural \int_{\tilde{G}} \Phi(\tilde{g}) T(\tilde{g}) d \tilde{g}=\int_{\tilde{G}}(L(x) \Phi(\tilde{g})) T(\tilde{g}) d \tilde{g} \quad\left(x \in \mathfrak{g}, \Phi \in C_{c}^{\infty}(\tilde{G})\right),
$$

where the integrals converge in the topology of $S^{*}\left(\mathfrak{s}_{1}\right)$.

Suppose the function $\Phi$ is supported in $-\tilde{G}^{c}=\tilde{c}_{-}\left(\mathfrak{g}^{c}\right)$. Then, by (6.15),

$$
T(x) \natural \int_{\tilde{G}} \Phi(\tilde{g}) T(\tilde{g}) d \tilde{g} \natural T(\tilde{c}(0))=\int_{\tilde{G}} L(x) \Phi(\tilde{g}) T(\tilde{g}) d \tilde{g} \natural T(\tilde{c}(0)),
$$

so that

$$
T(x) \natural \int_{\tilde{G}} \Phi\left(\tilde{g} \tilde{c}(0)^{-1}\right) T(\tilde{g}) d \tilde{g}=\int_{\tilde{G}} L(x) \Phi\left(\tilde{g} \tilde{c}(0)^{-1}\right) T(\tilde{g}) d \tilde{g},
$$


and therefore

$$
\begin{aligned}
& T(x) \downarrow \int_{\mathfrak{g}} \Phi \circ \tilde{c}_{-}(y) \Theta \circ \tilde{c}(y)\left|a_{\mathfrak{g}}(y)\right|^{-r_{\mathfrak{g}}} \chi_{y} d y \\
& =\int_{\mathfrak{g}}(L(x) \Phi) \circ \tilde{c}_{-}(y) \Theta \circ \tilde{c}(y)\left|a_{\mathfrak{g}}(y)\right|^{-r_{\mathfrak{g}}} \chi_{y} d y .
\end{aligned}
$$

Let $\phi=\Phi \circ \tilde{c}_{-}$. Then $\phi \in C_{c}^{\infty}\left(\mathfrak{g}^{c}\right)$ and, by (6.14), the above equation may be rewritten as

$$
\begin{aligned}
& T(x) \downarrow \int_{\mathfrak{g}} \phi(y)\left|a_{\mathfrak{g}}(y)\right|^{d^{\prime} / 2-r_{\mathfrak{g}}} \chi_{y} d y \\
& =\int_{\mathfrak{g}}\left(L(x)\left(\phi \circ \tilde{c}_{-}^{-1}\right)\right) \circ \tilde{c}_{-}(y)\left|a_{\mathfrak{g}}(y)\right|^{d^{\prime} / 2-r_{\mathfrak{g}}} \chi_{y} d y .
\end{aligned}
$$

Notice that

$$
\left(L(x)\left(\phi \circ \tilde{c}_{-}^{-1}\right)\right) \circ \tilde{c}_{-}=\left(L(x)\left(\phi \circ c_{-}^{-1}\right)\right) \circ c_{-} .
$$

Hence,

$$
T(x) \downarrow \int_{\mathfrak{g}} \phi(y)\left|a_{\mathfrak{g}}(y)\right|^{d^{\prime} / 2-r_{\mathfrak{g}}} \chi_{y} d y=\int_{\mathfrak{g}} c_{-}^{*}(L(x)) \phi(y)\left|a_{\mathfrak{g}}(y)\right|^{d^{\prime} / 2-r_{\mathfrak{g}}} \chi_{y} d y .
$$

In terms of the Fourier transform (2.8), with the form $B$ as in (6.9) and the map $\tau_{\mathfrak{g}}$ as in (1.17), the last equation may be rewritten as

$$
T(x) \llbracket\left(\mathcal{F}\left(\phi\left|a_{\mathfrak{g}}(y)\right|^{d^{\prime} / 2-r_{\mathfrak{g}}}\right) \circ \tau_{\mathfrak{g}}\right)=\mathcal{F}\left(\left(c_{-}^{*}(L(x)) \phi\right)\left|a_{\mathfrak{g}}(y)\right|^{d^{\prime} / 2-r_{\mathfrak{g}}}\right) \circ \tau_{\mathfrak{g}} .
$$

By (5.3), the right hand side of the above equation coincides with

$$
\mathcal{F}\left(L_{r_{\mathfrak{g}}-d^{\prime} / 2}(x) \phi\left|a_{\mathfrak{g}}(y)\right|^{d^{\prime} / 2-r_{\mathfrak{g}}}\right) \circ \tau_{\mathfrak{g}} .
$$

Thus we have shown that

$$
T(x) \llbracket\left(\mathcal{F}(\phi) \circ \tau_{\mathfrak{g}}\right)=\left(\hat{L}_{r_{\mathfrak{g}}-d^{\prime} / 2}(x) \mathcal{F} \phi\right) \circ \tau_{\mathfrak{g}} \quad\left(\phi \in C_{c}^{\infty}\left(\mathfrak{g}^{c}\right), x \in \mathfrak{g}\right) .
$$

Let $\psi$ be a polynomial function on $\mathfrak{g}$, homogeneous of degree $m$. Thus $\psi(t x)=$ $t^{m} \psi(x), t>0, x \in \mathfrak{g}$. Let $\phi \in C_{c}^{\infty}\left(\mathfrak{g}^{c}\right)$ be such that $\mathcal{F} \phi(0)=1$. Then

$$
\lim _{t \rightarrow 0} t^{-m} \psi(t x) \mathcal{F} \phi(t x)=\psi(x) \quad(x \in \mathfrak{g}) .
$$

Hence for any polynomial differential operator $D \in \mathcal{P} \mathcal{D}(\mathfrak{g})$,

$$
\lim _{t \rightarrow 0} t^{-m} D(\psi(t x) \mathcal{F} \phi(t x))=D \psi(x) \quad(x \in \mathfrak{g}) .
$$

Notice that $\mathcal{F} \phi(t x)$ is the Fourier transform of the function $t^{-\operatorname{dim}(\mathfrak{g})} \phi\left(t^{-1} x\right)$, whose support is equal to $t \operatorname{supp}(\phi)$, which is contained in $\mathfrak{g}^{c}$ if $\operatorname{supp} \phi$ is contained in a small ball near $0 \in \mathfrak{g}^{c}$ and if $t \leq 1$. Notice also that $\psi \circ \tau_{\mathfrak{g}}$ is a polynomial on $\mathfrak{s}_{1}$, homogeneous of degree $2 m$. Hence

$$
\lim _{t \rightarrow 0} t^{-m} \psi \circ \tau_{\mathfrak{g}}\left(t^{1 / 2} x\right)(\mathcal{F} \phi) \circ \tau_{\mathfrak{g}}\left(t^{1 / 2} x\right)=\psi \circ \tau_{\mathfrak{g}}(x) \quad\left(x \in \mathfrak{s}_{1}\right),
$$

and for any $D^{\prime} \in \mathcal{P} \mathcal{D}\left(\mathfrak{s}_{1}\right)$

$$
\lim _{t \rightarrow 0} t^{-m} D^{\prime}\left(\psi \circ \tau_{\mathfrak{g}}\left(t^{1 / 2} x\right)(\mathcal{F} \lambda) \circ \tau_{\mathfrak{g}}\left(t^{1 / 2} x\right)\right)=D^{\prime}\left(\psi \circ \tau_{\mathfrak{g}}\right)(x) \quad\left(x \in \mathfrak{s}_{1}\right) .
$$

Therefore (6.16) implies

$$
T(x) \natural\left(\psi \circ \tau_{\mathfrak{g}}\right)=\left(\hat{L}_{r_{\mathfrak{g}}-d^{\prime} / 2}(x) \psi\right) \circ \tau_{\mathfrak{g}} \quad(\psi \in \mathcal{P}(\mathfrak{g}), x \in \mathfrak{g}) .
$$

Let $E \subseteq \mathfrak{s}_{1}$ be a compact set. Then $K=\tau_{\mathfrak{g}}(E)$ is a compact subset of $\mathfrak{g}$. 
Let $\psi \in C^{\infty}(\mathfrak{g})$. By a version of the Weierstrass polynomial approximation theorem (see Appendix A), there is a sequence $\psi_{m} \in \mathcal{P}(\mathfrak{g})$ such that

$$
\lim _{m \rightarrow \infty} \max \left\{\left|D \psi(y)-D \psi_{m}(y)\right| ; y \in K\right\}=0 \quad(D \in \mathcal{P} \mathcal{D}(\mathfrak{g})) .
$$

Then by the chain rule

$$
T(x) \natural\left(\psi \circ \tau_{\mathfrak{g}}\right)(y)=\lim _{m \rightarrow \infty} T(x) \natural\left(\psi_{m} \circ \tau_{\mathfrak{g}}\right)(y) \quad(y \in E) .
$$

Since, by $(6.17)$,

and since

$$
T(x) \mathfrak{\natural}\left(\psi_{m} \circ \tau_{\mathfrak{g}}\right)=\left(\hat{L}_{r_{\mathfrak{g}}-d^{\prime} / 2}(x) \psi_{m}\right) \circ \tau_{\mathfrak{g}}
$$

$$
\lim _{m \rightarrow \infty} T(x) \mathfrak{b}\left(\psi_{m} \circ \tau_{\mathfrak{g}}\right)(y)=\left(\hat{L}_{r_{\mathfrak{g}}-d^{\prime} / 2}(x) \psi\right) \circ \tau_{\mathfrak{g}}(y) \quad(y \in E),
$$

by the above-mentioned Weierstrass theorem, the desired equation follows:

$$
T(x) \mathfrak{\natural}\left(\psi \circ \tau_{\mathfrak{g}}\right)=\left(\hat{L}_{r_{\mathfrak{g}}-d^{\prime} / 2}(x) \psi\right) \circ \tau_{\mathfrak{g}} \quad\left(\psi \in C^{\infty}(\mathfrak{g}), x \in \mathfrak{g}\right) .
$$

\section{Descent to A neighborhood of A SEMisimple Point \\ IN THE SYMPLECTIC SPACE $\mathfrak{s}_{1}$}

Let $x \in \mathfrak{s}_{1}$ and let $S^{x}$ be the stabilizer of $x$ in $S$.

Definition 7.1. A connected submanifold $U \subseteq \mathfrak{s}_{1}$ is called an admissible slice through $x$ if and only if

(1) $x \in U$,

(2) $U$ is $S^{x}$-stable,

(3) the tangent space $T_{x}\left(\mathfrak{s}_{1}\right)=T_{x}(U) \oplus T_{x}(S x)$,

(4) if $g \in S$ and $u, u^{\prime} \in U$ are such that $g u=u^{\prime}$, then $g \in S^{x}$, and

(5) the map $S \times U \ni(g, u) \rightarrow g u \in \mathfrak{s}_{1}$ is a submersion.

Condition (7.1.4) implies that the map

$$
\mu: S U \ni g u \rightarrow g x \in S x
$$

is well defined. As shown in $[\mathrm{V}, \mathrm{Vol} \mathrm{I}$, pages 15,16$], \mu$ is a locally trivial fibration with fiber $U$. In other words, for every point $g x \in S x$ there is an open neighborhood $M \subseteq S x$, and a diffeomorphism $\Phi$ such that the following diagram commutes:

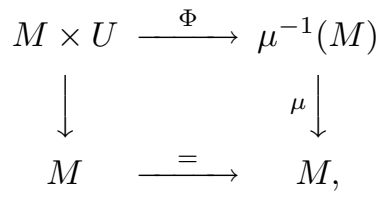

where the left vertical arrow is the projection on the first component.

Theorem $7.4([\mathrm{P} 0])$. Let $x \in \mathfrak{s}_{1}$ be semisimple. Then $\mathfrak{s}_{1}^{x}=\left\{y \in \mathfrak{s}_{1} ;[x, y]=0\right\}$, the commutator of $x$ in $\operatorname{End}(V)$ intersected with $\mathfrak{s}_{1}$, has a basis for the $S^{x}$-invariant neighborhoods of $x$ consisting of admissible slices $U_{x}$ through $x$, such that the maps

$$
\begin{aligned}
\tau_{\mathfrak{g}}: U_{x} & \rightarrow \mathfrak{g}^{x^{2}}, \\
\tau_{\mathfrak{g}^{\prime}}: U_{x} & \rightarrow \mathfrak{g}^{\prime x^{2}}
\end{aligned}
$$

are (injective) immersions. Moreover, the subset $\tau_{\mathfrak{g}^{\prime}}\left(U_{x}\right) \subseteq \mathfrak{g}^{\prime x^{2}}$ is open. (Recall that we assume the rank of $\mathfrak{g}^{\prime}$ to be less than or equal to the rank of $\mathfrak{g}$.) 
Let $\mathcal{U}\left(\mathfrak{g}_{\mathbb{C}}^{\prime}\right) \ni z \rightarrow \check{z} \in \mathcal{U}\left(\mathfrak{g}_{\mathbb{C}}^{\prime}\right)$ be the anti-involution such that $\check{z}=-z$ for $z \in \mathfrak{g}^{\prime}$. Let $R$ be the right regular representation

$$
R(x) \Psi(g)=\left.\frac{d}{d t} \Psi(g \exp (t x))\right|_{t=0} \quad\left(x \in \mathfrak{g}^{\prime}, g \in G^{\prime}, \Psi \in C^{\infty}\left(G^{\prime}\right)\right) .
$$

As in (2.7) and (2.11), define

$$
R_{p}(z)=c_{-}^{*}(R(z))+\tilde{p} z \quad \text { and } \quad \hat{R}_{p}(z)=R_{p}(z)^{r} \quad\left(z \in \mathfrak{g}^{\prime}, p \in \mathbb{C}\right) .
$$

Recall the notion of the pullback $\tau^{*}(u)$ of a distribution $u$ via a submersion $\tau$, [Hö, Theorem 6.1.2].

Corollary 7.7. For any $S$-invariant distribution $f$ defined on the set $\mathfrak{s}_{1}^{s s}$ of semisimple elements of $\mathfrak{s}_{1}$, there is a unique $G$-invariant distribution $f_{\mathfrak{g}}$ on $\tau_{\mathfrak{g}}\left(\mathfrak{s}_{1}^{s s}\right)$ and a unique $G^{\prime}$-invariant distribution $f_{\mathfrak{g}^{\prime}}$ on $\tau_{\mathfrak{g}^{\prime}}\left(\mathfrak{s}_{1}^{\text {ss }}\right)$ such that

$$
f=\tau_{\mathfrak{g}}^{*}\left(f_{\mathfrak{g}}\right)=\tau_{\mathfrak{g}^{\prime}}^{*}\left(f_{\mathfrak{g}^{\prime}}\right) .
$$

Moreover, for any $z \in \mathcal{U}\left(\mathfrak{g}_{\mathbb{C}}\right)^{G}$,

$$
f\left\lfloor T(z)=\tau_{\mathfrak{g}^{\prime}}^{*}\left(\hat{R}_{\mathfrak{g}^{\prime},-d / 2}\left(\mathcal{C}(z)^{\prime}\right) f_{\mathfrak{g}^{\prime}}\right)=f\lfloor T(\mathcal{C}(z)) .\right.
$$

Proof. Fix a point $x \in \mathfrak{s}_{1}^{s s}$, and let $U \subseteq \mathfrak{s}_{1}^{x}$ be an admissible slice through $x$. Let $\phi \in C_{c}^{\infty}(S U)$. Then there are $\psi \in C_{c}^{\infty}\left(\tau_{\mathfrak{g}}(U)\right)$ and $\psi^{\prime} \in C_{c}^{\infty}\left(\tau_{\mathfrak{g}^{\prime}}(U)\right)$ such that

$$
\int_{S^{x}} \phi(g y) d g=\psi \circ \tau_{\mathfrak{g}}(y)=\psi^{\prime} \circ \tau_{\mathfrak{g}^{\prime}}(y) \quad(y \in S U) .
$$

By the usual partition of unity argument, this implies (a). Furthermore, by (6.11), for any $z \in \mathcal{U}\left(\mathfrak{g}_{\mathbb{C}}\right)^{G}$ we have

$$
\begin{aligned}
& \int_{S^{x}}(T(z) \natural \phi)(g y) d g=T(z) \natural \int_{S^{x}} \phi(g y) d g \\
& =T(z) \natural\left(\psi^{\prime} \circ \tau_{\mathfrak{g}^{\prime}}\right)(y)=\left(\hat{L}_{\mathfrak{g}^{\prime}, r_{\mathfrak{g}^{\prime}}-d / 2}(\mathcal{C}(z)) \psi^{\prime}\right) \circ \tau_{\mathfrak{g}^{\prime}}(y) .
\end{aligned}
$$

For a vector space $W$ over $\mathbb{R}$, define an anti-involution $D \rightarrow D^{t}$ of the algebra $\mathcal{P} \mathcal{D}(W)$ by

$$
\partial(w)^{t}=-\partial(w), \quad q^{t}=q \quad(w \in W, q \in \mathcal{P}(W)),
$$

where $q$ is viewed as the multiplication operator $\phi \rightarrow q \phi$. Then the action of a differential operator $D$ on a distribution $f$ is given by $D(f)(\phi)=u\left(D^{t} \phi\right)$, Definition 3.1.1]. Equivalently, we have $D^{t}(f)(\phi)=u(D \phi)$. It is clear from (6.8.c) that

$$
(T(x) \natural)^{t}=\curvearrowleft T(x) \quad(x \in s p) .
$$

As in (2.3) we compute

$$
c_{-}^{*}(R(z))=\partial\left(\frac{1}{2}(y+1) z(-y+1)\right) \quad\left(z, y \in \mathfrak{g}^{\prime}\right) .
$$

A straightforward computation, using (7.9), shows that

$$
\hat{L}_{\mathfrak{g}^{\prime}, p}(z)^{t}=\hat{R}_{\mathfrak{g}^{\prime}, p-r_{\mathfrak{g}^{\prime}}}(-z) \quad\left(z \in \mathfrak{g}^{\prime}, p \in \mathbb{C}\right) .
$$

Hence,

$$
\begin{aligned}
& (f \llbracket T(z))(\phi)=(\llbracket T(z))^{t} f(\phi)=f(T(z) \natural \phi) \\
& =f_{\mathfrak{g}^{\prime}}\left(\hat{L}_{\mathfrak{g}^{\prime}, r_{\mathfrak{g}^{\prime}}-d / 2}(\mathcal{C}(z)) \psi^{\prime}\right)=\left(\hat{R}_{\mathfrak{g}^{\prime},-d / 2}(\mathcal{C}(z)) f_{\mathfrak{g}^{\prime}}\right)\left(\psi^{\prime}\right),
\end{aligned}
$$

and (b) follows. 


\section{Appendix A. The Weierstrass polynomial approximation theorem}

Lemma. For any function $\psi \in C^{\infty}\left(\mathbb{R}^{n}\right)$ and any compact set $K \subseteq \mathbb{R}^{n}$ there is a sequence of polynomials $\psi_{m} \in \mathcal{P}\left(\mathbb{R}^{n}\right)$ such that

$$
\lim _{m \rightarrow \infty} \max \left\{\left|D \psi(x)-D \psi_{m}(x)\right| ; x \in K\right\}=0 \quad\left(D \in \mathcal{P} \mathcal{D}\left(\mathbb{R}^{n}\right)\right) .
$$

Proof. We trace through the proof of Theorem 4.5 in [B] in the case $n=1$. The general case is entirely analogous.

We may assume that supp $\psi \subseteq(-\pi, \pi)$ and $K \subseteq(-\pi, \pi)$. Also, we may assume that $D$ is a differential operator with constant coefficients.

As is well known, there is a sequence of trigonometric polynomials

$$
t_{m}(x)=\sum_{k} a_{k, m} e^{i k x} \quad(x \in \mathbb{R})
$$

such that for any $n=0,1,2, \ldots$,

$$
\lim _{m \rightarrow \infty}\left\|\psi^{(n)}-t_{m}^{(n)}\right\|_{\infty}=0 .
$$

Let

$$
\psi_{m}(x)=\sum_{k} a_{k, m} \sum_{d=0}^{m} \frac{(i k x)^{d}}{d !} .
$$

Then

$$
t_{m}^{(n)}(x)-\psi_{m}^{(n)}(x)=\sum_{k} a_{k, m} \sum_{d=m+1-n}^{m}(i k)^{n} \frac{(i k x)^{d}}{d !} \quad(x \in \mathbb{R}) .
$$

Hence for $|x| \leq 1$,

$$
\left|t_{m}^{(n)}(x)-\psi_{m}^{(n)}(x)\right| \leq \sum_{k}\left|a_{k, m}\right| \sum_{d=m+1-n}^{m} k^{n} \frac{(k \pi)^{d}}{d !} \quad(x \in \mathbb{R}) .
$$

Therefore,

$$
\lim _{m \rightarrow \infty} \max \left\{\left|t_{m}^{(n)}(x)-\psi_{m}^{(n)}(x)\right| ;|x| \leq 1\right\}=0 .
$$

Appendix B. Completion of the proof of Theorem 2.13

Here we verify the last statement of Theorem 2.13. We may assume that the group $G$ is compact. Let $\Psi \in C^{\infty}(G)$. For $z \in \mathcal{U}\left(\mathfrak{g}_{\mathbb{C}}\right)$ we have

$$
\int_{G} L(z) \Psi(g) d g=\varepsilon_{\mathfrak{g}}(z) \int_{G} \Psi(g) d g .
$$

Thus if $\Psi$ is supported in the domain of the Cayley transform $c_{-}$, then

$$
\int_{\mathfrak{g}}(L(z) \Psi) \circ c_{-}(y)\left|a_{\mathfrak{g}}(y)\right|^{-r_{\mathfrak{g}}} d y=\varepsilon_{\mathfrak{g}}(z) \int_{\mathfrak{g}} \Psi \circ c_{-}(y)\left|a_{\mathfrak{g}}(y)\right|^{-r_{\mathfrak{g}}} d y .
$$

Let $\psi=\Psi \circ c_{-}$. Then

$$
\int_{\mathfrak{g}} c_{-}^{*}(L(z)) \psi(y)\left|a_{\mathfrak{g}}(y)\right|^{-r_{\mathfrak{g}}} d y=\varepsilon_{\mathfrak{g}}(z) \int_{\mathfrak{g}} \psi(y)\left|a_{\mathfrak{g}}(y)\right|^{-r_{\mathfrak{g}}} d y .
$$

The left hand side is equal to

$$
\int_{\mathfrak{g}}\left|a_{\mathfrak{g}}(y)\right|^{-r_{\mathfrak{g}}} c_{-}^{*}(L(z))\left|a_{\mathfrak{g}}(y)\right|^{r_{\mathfrak{g}}}\left|a_{\mathfrak{g}}(y)\right|^{-r_{\mathfrak{g}}} \psi(y) d y .
$$


Hence if $f(y)=\psi \cdot\left|a_{\mathfrak{g}}(y)\right|^{-r_{\mathfrak{g}}}$, then

$$
\int_{\mathfrak{g}} \frac{1}{a_{\mathfrak{g}}^{r_{\mathfrak{g}}}} c_{-}^{*}(L(z)) a_{\mathfrak{g}}^{r_{\mathfrak{g}}} \cdot f(y) d y=\varepsilon_{\mathfrak{g}}(z) \int_{\mathfrak{g}} f(y) d y .
$$

Thus

$$
\int_{\mathfrak{g}} L_{\mathfrak{g}, r_{\mathfrak{g}}}(z) f(y) d y=\varepsilon_{\mathfrak{g}}(z) \int_{\mathfrak{g}} f(y) d y,
$$

and therefore

$$
\hat{L}_{\mathfrak{g}, r_{\mathfrak{g}}}(z) \hat{f}(0)=\varepsilon_{\mathfrak{g}}(z) \hat{f}(0) .
$$

\section{REFERENCES}

[C1] A. Capelli, Über die Zurückführung der Cayley'schen Operator $\Omega$ auf gewönliche PolarOperationen, Mathematische Annalen 29 (1887), 331-338.

[C2] A. Capelli, Sur les opérations dans la théorie des formes algébriques, Mathematische Annalen 37 (1890), 1-37.

[B] R. Beals, Advanced Mathematical Analysis, Springer-Verlag, 1987. MR 58:26624

[G-W] R. Goodman and N. Wallach, Representations and invariants of the classical groups., Cambridge University Press, 1998. MR 99b:20073

[H-C1] Harish-Chandra, The characters of semisimple Lie groups, Trans. Amer. Math. Soc. 83 (1956), 98-163. MR 18:318c

[H-C2] Harish-Chandra, Differential operators on a semisimple Lie algebra, Amer. J. Math. 79 (1957), 87-120. MR 18:809d

[H-C3] Harish-Chandra, Invariant differential operators and distributions on a semisimple Lie algebra, Amer. J. Math. 86 (1964), 534-564. MR 31:4862a

[H-C4] Harish-Chandra, Invariant eigendistributions on a semisimple Lie group, Trans. Amer. Math. Soc. 119 (1965), 457-508. MR 31:4862d

[H-C5] Harish-Chandra, Invariant distributions on Lie algebras, Amer. J. of Math. 86 (1964), 271-309. MR 28:5144

[H0] R. Howe, Remarks on classical invariant theory, Trans. Amer. Math. Soc. 313 (1989), 539-570. MR 90h:22015a, MR 90h:22015b

[H1] R. Howe, Transcending classical invariant theory, J. Amer. Math. Soc. 2 (1989), 535-552. MR 90k:22016

[H2] R. Howe, The oscillator semigroup, Proc. Symp. Pure Math., Vol. 48, Amer. Math. Soc.: Providence, 1988, pp. 61-132. MR 90f:22014

[H-U] R. Howe and T. Umeda, The Capelli identity, the double commutant theorem, and multiplicity free actions, Mathematische Annalen 290 (1991), 565-619. MR 92j:17004

[Hö] L. Hörmander, The analysis of linear partial differential operators, I, Springer Verlag, 1983. MR 85g:35002a

[I] M. Itoh, Capelli identities for the dual pair $\left(O_{M}, S p_{N}\right)$, preprint.

[J] N. Jacobson, Basic algebra I. Second edition., W. H. Freeman and Company, New York, 1985. MR 86d:00001

[K] V. Kac, Lie superalgebras, Advances in Math. 26 (1977), 8-96. MR 58:5803

[Ko] B. Kostant, Graded manifolds, graded Lie theory, and prequantization, Lecture Notes in Math., 570 Springer-Verlag, Berlin-New York 1977, pp. 177-306. MR 58:28326

[L-S1] T. Levasseur and J. T. Stafford, Rings of differential operators on classical rings of invariants, Mem. Amer. Math. Soc. 81 (1989). MR 90i:17018

[L-S2] T. Levasseur and J. T. Stafford, Invariant differential operators and an homomorphism of Harish-Chandra, J. Amer. Math. Soc. 8 (1995), 365-372. MR 95g:22029

[L-S3] T. Levasseur and J. T. Stafford, The kernel of an homomorphism of Harish-Chandra, Ann. Sci. École Norm. Sup. 3 (1996), 385-397. MR 97b:22019

[Pr] V. Protsak, A theory of rank for enveloping algebras. I, preprint.

[P0] T. Przebinda, Local Geometry of Orbits for an Ordinary Classical Lie Supergroup, in preparation.

[P1] T. Przebinda, Characters, dual pairs, and unitary representations, Duke Math. J. 69 (1993), 547-592. MR 94i:22036 
[P2] T. Przebinda, The duality correspondence of infinitesimal characters, Colloq. Math $\mathbf{7 0}$ (1996), 93-102. MR 96m:22034

[Sm-St] S.P. Smith and J. T. Stafford, Differential operators on an affine curve, Proc. London Math. Soc. (3) 56 (1988), 229-259. MR 89d:14039

[V] V. S. Varadarajan, Harmonic analysis on real reductive groups, Lecture Notes in Mathematics, 576 Springer-Verlag, Berlin-New York 1977. MR 57:12789]

[W] N. R. Wallach, Invariant differential operators on a reductive Lie algebra and Weyl group representations, J. Amer. Math. Soc. 6 (1993), 779-816. MR 94a:17014

Department of Mathematics, University of Oklahoma, Norman, Oklahoma 73019

E-mail address: tprzebin@crystal.math.ou.edu 